\title{
The Mechanosensory Lateral Line System Mediates Activation of Socially-Relevant Brain Regions during Territorial Interactions
}

Julie M. Butler and Karen P. Maruska *

Department of Biological Sciences, Louisiana State University, Baton Rouge, LA, USA

Animals use multiple senses during social interactions and must integrate this information in the brain to make context-dependent behavioral decisions. For fishes, the largest group of vertebrates, the mechanosensory lateral line system provides crucial hydrodynamic information for survival behaviors, but little is known about its function in social communication. Our previous work using the African cichlid fish, Astatotilapia burtoni, provided the first empirical evidence that fish use their lateral line system to detect water movements from conspecifics for mutual assessment and behavioral choices. It is unknown, however, where this socially-relevant mechanosensory information is processed in the brain to elicit adaptive behavioral responses. To examine for the first time in any fish species which brain regions receive contextual mechanosensory information, we quantified expression of the immediate early gene cfos as a proxy for neural activation in sensory and socially-relevant brain nuclei from lateral line-intact and -ablated fish following territorial interactions. Our in situ hybridization results indicate that in addition

OPEN ACCESS

Edited by:

Nuno Sousa,

University of Minho, Portugal

Reviewed by:

D. Caroline Blanchard, University of Hawaii at Manoa, USA

Rui F. Oliveira,

ISPA - Instituto Universitário, Portugal

*Correspondence:

Karen P. Maruska

kmaruska@/su.edu

Received: 19 December 2015 Accepted: 29 April 2016 Published: 13 May 2016

Citation:

Butler JM and Maruska KP (2016) The Mechanosensory Lateral Line System

Mediates Activation of

Socially-Relevant Brain Regions during Territorial Interactions.

Front. Behav. Neurosci. 10:93. doi: 10.3389/fnbeh.2016.00093 to known lateral line processing regions, socially-relevant mechanosensory information is processed in the ATn (ventromedial hypothalamus homolog), DI (putative hippocampus homolog), and Vs (putative medial extended amygdala homolog). In addition, we identified a functional network within the conserved social decision-making network (SDMN) whose co-activity corresponds with mutual assessment and behavioral choice. Lateral line-intact and -ablated fight winners had different patterns of co-activity of these function networks and group identity could be determined solely by activation patterns, indicating the importance of mechanoreception to co-activity of the SDMN. These data show for the first time that the mechanosensory lateral line system provides relevant information to conserved decision-making centers of the brain during territorial interactions to mediate crucial behavioral choices such as whether or not to engage in a territorial fight. To our knowledge, this is also the first evidence of a subpallial nucleus receiving mechanosensory input, providing important information for elucidating homologies of decision-making circuits across vertebrates. These novel results highlight the importance of considering multimodal sensory input in mediating context-appropriate behaviors that will provide broad insights on the evolution of decision-making networks across all taxa.

Keywords: assessment, cichlid, mechanoreception, sensory processing, social behavior, social decision-making, teleost 


\section{INTRODUCTION}

Sensory signals from conspecifics provide relevant and necessary information for animals to make context-appropriate behavioral decisions. The social behavior network (SBN) is a group of conserved brain nuclei that possess sex steroid receptors, are reciprocally connected, and are involved in a variety of social behaviors, such as aggression, courtship, spawning, and parental care (Newman, 1999; Goodson, 2005). The SBN, in conjunction with the mesolimbic reward system, comprise the conserved social decision-making network (SDMN), which is thought to evaluate the salience of social cues to produce suitable behavioral responses (O'Connell and Hofmann, 2011). Many of the nuclei (or nodes) of the SDMN are connected with sensory processing regions but the relative role of inputs from different sensory modalities in mediating behavioral responses remains relatively unexplored, particularly in teleost fishes, the largest, and most diverse group of vertebrates.

The teleost mechanosensory lateral line system allows fish to detect near flow water movements (Dijkgraaf, 1963), such as those created by water currents, approaching predators, or conspecifics during social interactions (reviewed in Montgomery et al., 2014). Lateral line-mediated vibrational communication is used by several fish species during reproductive and spawning behaviors (Satou et al., 1994; Mirjany et al., 2011; Medina et al., 2013), and the African cichlid fish Astatotilapia burtoni uses hydrodynamic cues during territorial interactions (Butler and Maruska, 2015). Mechanosensory cues are intentionally produced by many fish species as a form of communication (Satou et al., 1994; Mirjany et al., 2011; Medina et al., 2013; Butler and Maruska, 2015), and without a functioning lateral line system, fish have altered behaviors and are less likely to engage in social interactions. For example, lateral line-ablated A. burtoni dominant males were $\sim 50 \%$ less likely than lateral line-intact males to engage in a territorial interaction (Butler and Maruska, 2015), and lateral line-ablated hime salmon males were less likely to display courtship and spawning behaviors (Satou et al., 1994). In addition, the A. burtoni lateral line

\footnotetext{
Abbreviations: ATn, anterior tuberal nucleus; ANOVA, analysis of variance; BM, body mass; CC, cerebellar crest; CDA, canonical discriminant function analysis; $\mathrm{CoCl}_{2}$, cobalt chloride; CP, central posterior thalamic nucleus; DASPEI, 2-[4(dimethylamino)styryl]- $N$-ethylpyridinium iodide; Dc, central part of the dorsal telencephalon; Dc-4, Dc, subdivision 4; Dl, lateral zone of the dorsal telencephalon; Dlg, granular zone of Dl; Dm, medial part of the dorsal telencephalon; Dp, posterior portion of the dorsal telencephalon; E, entopeduncular nucleus; EGTA, ethylene glycolbis tetraacetic acid; GSI, gonadosomatic index; ICL, inner cellular layer of the OB; MON, medial octavolateralis nucleus; NLT, lateral tuberal nucleus; nMMp, magnocellular preoptic nucleus, magnocellular division; $\mathrm{nPMp}$, magnocellular preoptic nucleus, parvocellular division; OB, olfactory bulb; PAG, periaqueductal gray; PCA, principal component analysis; Pit, pituitary; PGl, lateral preglomerular nucleus; PTT, paratoral tegmental nucleus; SBN, social behavior network; SDMN, social decision-making network; SL, standard length; TPp, periventricular nucleus of the posterior tuberculum; TSc, central portion of the torus Semicircularis; TSvl, ventrolateral portion of the torus Semicircularis; $\mathrm{Vc}$, central part of the ventral telencephalon; VCeg, granular layer of vulvula cerebellum; Vd, dorsal part of the ventral telencephalon; Vdc, caudal subdivision of $\mathrm{Vd}$; $\mathrm{Vl}$, lateral zone of the ventral telencephalon; $\mathrm{Vp}$, postcommissural nucleus of the ventral telencephalon; Vs, supracommissural nucleus of the ventral telencephalon; VTn, ventral tuberal nucleus; Vv, ventral part of the ventral telencephalon.
}

system is used to assess opponents and facilitates the use of less dangerous non-contact behaviors over contact behaviors when engaged in male-male territorial contests (Butler and Maruska, 2015). Despite the known importance of the lateral line system in mediating these reproductive and territorial interactions, it remains unknown where this socially-relevant mechanosensory information is processed in the brain to facilitate behavioral decisions.

The highly social African cichlid fish A. burtoni is an ideal model system to examine the role of hydrodynamic information in mediating activation of socially-relevant brain regions. A. burtoni have a diverse behavioral repertoire (Fernald, 1977; Fernald and Hirata, 1977) that produces a variety of multimodal sensory signals during social interactions (visual: Chen and Fernald, 2011, chemosensory: Maruska and Fernald, 2012, auditory: Maruska et al., 2012b, mechanosensory: Butler and Maruska, 2015). Males exist as two distinct phenotypes, dominant and subordinate, and can rapidly switch between the two depending on their social environment, with rapid consequences for their reproductive physiology (Maruska and Fernald, 2013; Maruska et al., 2013; Maruska, 2014). Dominant males are brightly-colored, actively court females, and defend their spawning territory from other males (Fernald, 1977; Fernald and Hirata, 1977), and many of these behaviors involve fin and body movements that produce hydrodynamic signals detected by the lateral line system of near-by fish. But where in the brain are these socially-relevant hydrodynamic signals processed to produce behavioral changes?

Although not previously investigated, it is possible that the lateral line system provides relevant information to the SDMN to influence behavioral output during social interactions. By quantifying brain activation using an immediate early gene ( $c f o s$ ) as a proxy for neural activation, we identify for the first time in any fish species which brain regions are important for processing socially-relevant mechanosensory information in a territorial context. We first examined individual nodes of the SDMN to investigate whether these regions received mechanosensory input, and then combined activation data to examine the role of mechanosensory information on functional connectivity and co-activation across the SDMN.

\section{MATERIALS AND METHODS}

\section{Experimental Animals}

Adult A. burtoni were bred from a wild-caught stock from Lake Tanganyika, Africa, and were maintained in an environment that mimicked their natural habitat. A. burtoni were housed in $30 \mathrm{~L}$ aquaria at $28-30^{\circ} \mathrm{C}$ on a $12 \mathrm{~L}: 12 \mathrm{D}$ cycle and fed cichlid flakes (AquaDine, Healdsburg, CA) once daily and supplemented with brine shrimp. All experiments were performed in accordance with the recommendations and guidelines stated in the National Institutes of Health (NIH) Guide for the Care and Use of Laboratory Animals, 2011. The protocol was approved by the Institutional Animal Care and Use Committee (IACUC) at Louisiana State University, Baton Rouge, LA. The animals used in this experiment are a subset of animals used in previously published behavioral analyses of the role of the mechanosensory 
lateral line system during male-male territorial interactions (Butler and Maruska, 2015).

\section{Behavior Experiments}

We previously demonstrated that the mechanosensory lateral line system in A. burtoni is used during territorial interactions (Butler and Maruska, 2015). Here, we used the same paradigm as previously described in which dominant male fish were forced to compete over a novel territory (Figure 1A). A single 10-gallon tank was divided into two equal compartments $(25.4 \times 31.2 \times$ $25.4 \mathrm{~cm}$ ) by a removable opaque acrylic barrier and a quarter terra-cotta pot was placed on either side of the barrier to serve as a territory for each subject fish. Experimental male fish were chosen based on their displays of typical dominance behaviors in community tanks for $\sim 5$ days before being moved to the experimental tank to acclimate for 2 days (including treatment time) in their new territory. Each animal was used for only one behavior trial. Animals were size-matched for both SL (41.000 $\pm 4.283 \mathrm{~mm}$, mean $\pm \mathrm{SD})$ and $\mathrm{BM}(2.128 \pm 1.828 \mathrm{~g})$ so that no fish was more than $10 \%$ larger than his opponent, and to ensure that SL and BM did not differ between trial pairings (SL: MannWhitney Rank Sum: $U=69.000, T=153.000, P=0.884$; BM: $U=45.000, T=177.000, P=0.126$ ).

On the morning of the experimental trial, fish were allowed to acclimate to the video camera for $\sim 10$ min before the camera was turned on to record a 5-min pre-trial behavior baseline. The opaque barrier was then removed and the two quarter pots were repositioned to form a single territory shelter. In this paradigm, the two dominant males with individual territories are then forced to fight over the new single territory shelter in the same tank and have equal opportunity to acquire the territory. During the trial time, opaque barriers were placed on either end of the tank to block the view of other fish in adjacent tanks.

All trials were recorded and later quantified for prefight and fight behaviors, including contact (one fish physically touching the other) and non-contact (within one body length but not physically touching) behaviors. Non-contact behaviors included lateral displays (fish orient parallel or perpendicular to each other, erect fins, distend jaws, and shake their bodies), lunges (quick movement toward opponent without physical contact), and frontal threats (fish distends jaw and flares opercula while moving toward opponent). Contact behaviors included abnormal lateral displays (lateral display but with physical contact to the opponent), mouth fights (opponents grasp one another by the mouth and push, pull, and turn), bites (with mouth open, one fish makes physical contact with the opponent), and nudges/rams (with mouth closed, one fish pushes the other). Fight onset was defined as the first reciprocal exchange of aggressive behaviors, and fights had to last a minimum of $30 \mathrm{~s}$. Fish were allowed to fight until a clear winner and loser was established based on the following criteria as outlined previously (Maruska et al., 2013; Butler and Maruska, 2015). The winner fish had to fulfill two of the following characteristics: (1) entered the shelter $>3$ times within a 1-min period, (2) entered and stayed in the shelter for $>10$ consecutive seconds, (3) performed at least 3 dominance behaviors within $1 \mathrm{~min}$, and (4) chased or bit the other male. The subordinate, or loser, fish had to fulfill both of the following criteria: (1) loss of eye bar and bright coloration, and (2) perform typical submissive behaviors (e.g., fleeing, hiding at the top of the water column). Latency to fight was defined as the time between removing the barrier and the fight onset, and fight duration as the time between fight onset and establishment of a winner. Assessment time was defined as the percent of prefight time spent within one body length of the opponent without performing an aggressive display.

\section{Mechanosensory Lateral Line Ablation}

To compare behavior of lateral line-intact and lateral line-ablated fish, experimental fish were randomly assigned to one of two groups prior to use in behavioral experiments described above: sham treatment or lateral line ablation. Lateral line ablation was done by immersing fish in cichlid-system fish water containing $2 \mathrm{mM}$ cobalt chloride hexahydrate $\left(\mathrm{CoCl}_{2}\right.$; Sigma) and $1 \mathrm{mM}$ EGTA (ethylene glycolbis tetraacetic acid; Sigma-Aldrich) for $3 \mathrm{~h}$. Immediately following $\mathrm{CoCl}_{2}$ treatment, fish were placed in ice water for $\sim 3 \mathrm{~min}$ before the posterior lateral line nerve was bilaterally transected. To cut the nerve, 2-3 scales were gently removed at the posterior dorsal edge of the operculum and a \#11 scalpel was used to make a small incision through the skin and superficial muscle about $4 \mathrm{~mm}$ in length. The posterior lateral line nerve (pLLn) was then visible and a $1-2 \mathrm{~mm}$ portion of the nerve was removed. Using a dose of $2 \mathrm{mM} \mathrm{CoCl}$ for $3 \mathrm{~h}$ consistently ablated neuromasts located on the head. This treatment, however, left neuromasts on the trunk and tail of the animals intact. To resolve this issue, we bilaterally clipped the pLLns to remove mechanosensory input from the remaining intact neuromasts on the trunk and tail. Together, these treatments removed lateral line input from both the cranium (due to $\mathrm{CoCl}_{2}$-treatment) and the trunk (pLLn transections). The efficacy of this treatment was previously established, and our previous study demonstrated no toxicity effects from the cobalt chloride treatment (Butler and Maruska, 2015).

Sham handled fish (i.e., lateral line-intact fish) were transferred to a similar-sized container as the ablated fish and immersed in normal cichlid-system fish water for $3 \mathrm{~h}$. Following treatment, fish were placed in ice water and a small incision was made into the dorsal musculature near the dorsal fin to insure that it did not affect the lateral line system. Sham treatment was used to control for handling stress (e.g., $3 \mathrm{~h}$ in beaker and minor surgery). Following treatment or shamhandling, fish were returned to their half of the experimental tank overnight, and behavior experiments took place $18 \mathrm{~h}$ after the end of treatment, a time during which neuromast function in the cobalt-treated fish did not recover (verified with DASPEI staining).

\section{Tissue Collection}

To compare brain activation patterns of lateral line-intact and ablated fish, we collected brains from six intact-intact pairs (i.e., six winners, six losers) and six ablated-ablated pairs. Fish were quickly removed from the experimental tank 30-min post fight (using the above criteria), anesthetized in ice cold fish water, measured for standard length (SL) and body mass (BM), and killed by rapid cervical transection. Brains were exposed and 
A

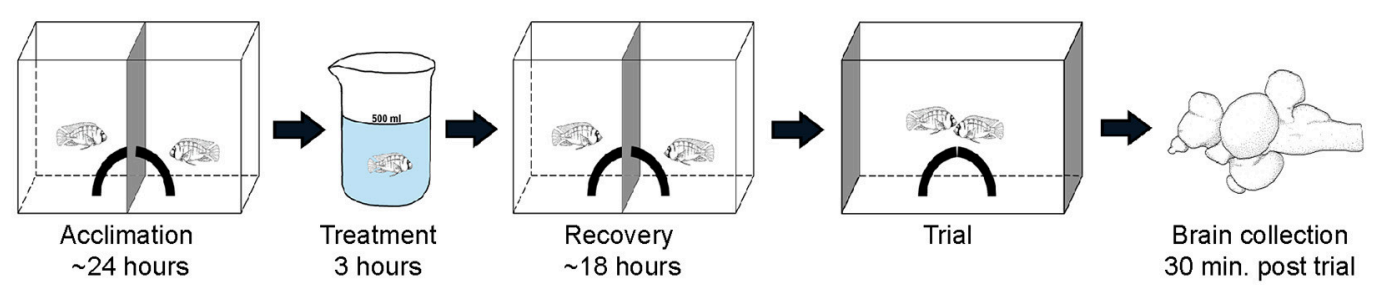

B

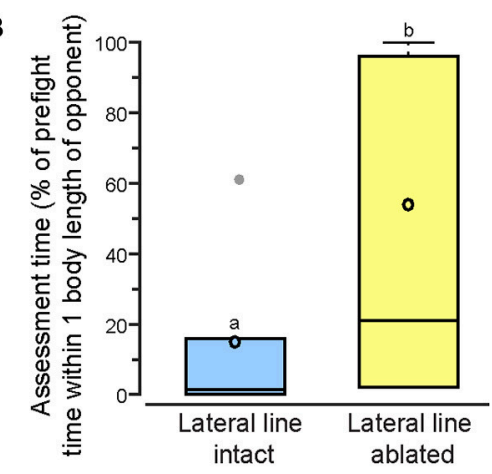

C

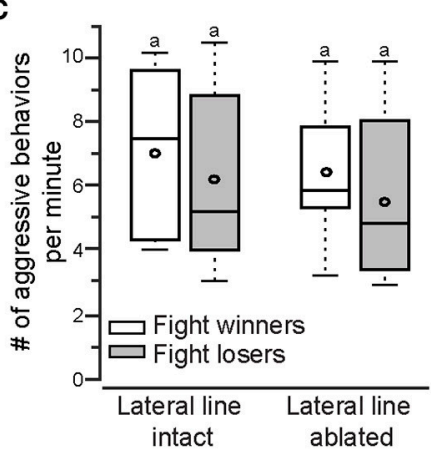

D

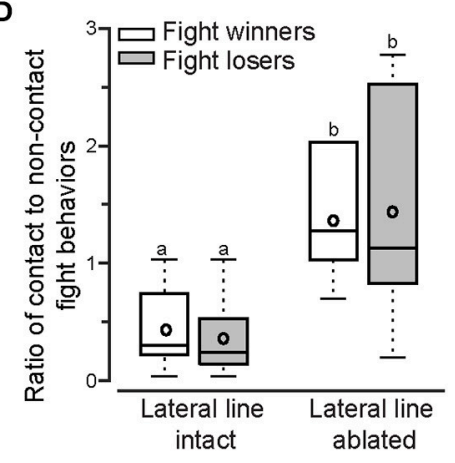

FIGURE 1 | Lateral line-ablated animals have decreased assessment abilities and prefer contact over non-contact fight behaviors. (A) Two dominant Astatotilapia burtoni males acclimated on either side of an opaque blue barrier for $24 \mathrm{~h}$ with a quarter of a terracotta pot to serve as a territory. Fish were treated for $3 \mathrm{~h}$ (intact: normal cichlid-system water; ablated: $2 \mathrm{mM}$ cobalt chloride in low calcium cichlid-system water) and placed back in the experimental tank to recover for $18 \mathrm{~h}$ prior to behavioral testing. Total acclimation time, including treatment, was $\sim 2$ days. On the day of the trial, the center barrier was removed and the two quarter pots repositioned to form a single half-pot territory to induce a territorial fight. Opaque barriers were placed on both ends of the tank to block the view of neighboring fish during trials. Animals were sacrificed and brains collected $30 \mathrm{~min}$ after the fight concluded. Modified from Butler and Maruska (2015). (B) Lateral line-ablated fish (yellow) had increased assessment time (quantified as the percent of pre-fight time spent within one body length of the opponent without performing an aggressive behavior) than lateral line-intact fish (blue). (C) The number of aggressive behaviors per minute was not different between lateral line-intact and lateral line-ablated fight winners and losers. (D) Lateral line-ablated fish used more contact than non-contact fight behaviors ( $>1$ signifies reliance on contact behaviors, $<1$ signifies preference for non-contact behaviors). Different letters indicate statistical significance at $P<0.05$. Tukey's box plots were used to represent behavior data $(N=6$ per group): data median is represented by a line and data mean by an open circle, the box extends to the furthest data points within the 25th and 75th percentile, and whiskers extend to the furthest data points in the 25th/75th percentiles. Absence of whiskers indicates absence of data points outside of the 25th/75th percentiles. Data points outside the 25 th/75th percentiles are represented by closed gray circles.

fixed in the head overnight at $4^{\circ} \mathrm{C}$ in $4 \%$ paraformaldehyde (PFA) in $1 \times$ phosphate-buffered saline $(1 \times \mathrm{PBS})$, rinsed for $24 \mathrm{~h}$ in $1 \times \mathrm{PBS}$, and cryoprotected overnight in $30 \%$ sucrose in $1 \times$ PBS. Gonads were removed and weighed (gonad mass, $\mathrm{GM})$ to calculate gonadosomatic index $[\mathrm{GSI}=(\mathrm{GM} / \mathrm{BM}) * 100$; $0.846 \pm 0.173]$. GSI did not differ among treatment groups $(t=$ $-0.083 ; d f=22 ; P=0.934)$. Brains were then embedded in OCT media (TissueTek, Sakura), sectioned in the transverse plane on a cryostat (Leica, CM1850) at $20 \mu \mathrm{m}$, and collected onto 2 alternate sets of charged slides (VWR superfrost plus). Slides were dried flat at room temperature for 2 days prior to storage at $-80^{\circ} \mathrm{C}$.

\section{Preparation of Dig-Labeled Riboprobe for In situ Hybridization}

The immediate early gene cfos is commonly used as a marker of neural activation. To visualize $c f o s$ mRNA in the brain, we used chromogenic in situ hybridization (ISH) with a riboprobe specific to the A. burtoni cfos mRNA sequence. Primers were designed based on the sequence available in Genbank (HQ232413.1) and commercially synthesized (Life Technologies; forward primer: $5^{\prime}$-agagaactgatcgggagcagcgct-3'; reverse primer: $5^{\prime}$-caggttgggatatcattctgcagg- $\left.3^{\prime}\right)$. Probe template was generated by PCR amplification (Platinum SuperMix, Life Technologies) of whole brain $A$. burtoni cDNA, cfos gene-specific primers, and the following reaction conditions: $95^{\circ} \mathrm{C}$ for $1 \mathrm{~min}, 45$ cycles of: $\left(95^{\circ} \mathrm{C}\right.$ for $15 \mathrm{~s}, 55^{\circ} \mathrm{C}$ for $15 \mathrm{~s}, 72^{\circ} \mathrm{C}$ for $1 \mathrm{~min}$ ), and $72^{\circ} \mathrm{C}$ for 1 min. A transcription reaction was used to incorporate DIG (DIGlabeling mix, Roche)-labeled nucleotides into the purified PCR template (MinElute PCR Kit, Qiagen) before probe purification (GE Illustra Probe Quant G-50 microcolumns). PCR products and the final probe were checked on a $1 \%$ agarose gel after each step and verified as a band of the correct size. The probe was then diluted 1:5 in hybridization buffer and stored at $-20^{\circ} \mathrm{C}$ until use. Probes were transcribed using the T3 polymerase transcription initiation sequence (aattaaccctcactaaaggg) that was added to the reverse (for anti-sense probes) or forward (for sense control probes) primer.

\section{In situ Hybridization}

In situ hybridization was performed to visualize and quantify brain activation differences between lateral line-intact and ablated fish following territorial interactions. To minimize inter-ISH variability for quantification, each staining reaction contained brains from individuals from each treatment group. Slides of cryosectioned brains were thawed to room temperature and a hydrophobic barrier (Immedge pen, Vector Laboratories) 
was applied around the sections and allowed to dry for $40 \mathrm{~min}$. Slides were incubated at room temperature in $1 \times$ PBS $(3 \times$ $5 \mathrm{~min}), 4 \%$ PFA $(20 \mathrm{~min}), 1 \times$ PBS $(2 \times 5 \mathrm{~min})$, proteinase

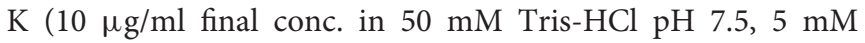
EDTA pH 8.0; $10 \mathrm{~min}), 1 \times$ PBS (10 min), 4\% PFA (15 min), $1 \times$ PBS $(2 \times 5 \mathrm{~min})$, milliQ water $(3 \mathrm{~min}), 0.25 \%$ pure acetic anhydride in $0.1 \mathrm{M}$ triethanolamine- $\mathrm{HCl} \mathrm{pH} 8.0$ (10 min), and $1 \times$ PBS $(5 \mathrm{~min})$. Slides were then placed in a sealed humidified chamber at $60-65^{\circ} \mathrm{C}$ and incubated in pre-warmed hybridization buffer without probe (50\% formamide, $5 \times$ SSC, $0.1 \%$ tween- 20 , $0.1 \%$ CHAPS, $5 \mathrm{mM}$ EDTA, $1 \mathrm{mg} / \mathrm{ml}$ torula RNA) for $3 \mathrm{~h}$. Slides were incubated with $c f o s$ riboprobe, sealed with hybrislips (Life Technologies) to evenly distribute probe and prevent drying, and placed in a sealed, humidified chamber in a hybridization oven at $60-65^{\circ} \mathrm{C}$. After $12-16 \mathrm{~h}$ of hybridization, hyrbislips were removed in pre-warmed $2 \times$ SSC:50\% formamide with $0.1 \%$ tween-20 solution and the following washes were performed at 60-65 ${ }^{\circ} \mathrm{C}: 2 \times$ SSC:formamide $(2 \times 30 \mathrm{~min}), 2 \times$ SSC:Maleate Buffer (MABT; $100 \mathrm{mM}$ maleic acid pH 7.2, $150 \mathrm{mM} \mathrm{NaCl}, 0.1 \%$ tween-20; $2 \times 15 \mathrm{~min})$, and MABT $(2 \times 10 \mathrm{~min})$. Slides were then washed at room temperature in MABT $(2 \times 10 \mathrm{~min})$, blocked in MABT with $2 \%$ bovine serum albumin ( $3 \mathrm{~h}$ ) at room temperature, and incubated in alkaline-phosphatase-conjugated anti-DIG Fab fragments (Roche; 1:5000 dilution in blocking solution) overnight $(12-16 \mathrm{~h})$ at $4^{\circ} \mathrm{C}$ in a humidified chamber. Slides were then rinsed in MABT at room temperature $(3 \times$ $30 \mathrm{~min})$, incubated in alkaline phosphate buffer $(2 \times 5 \mathrm{~min})$, and developed with nitro-blue tetrazolium/5-bromo-4-chloro$3^{\prime}$-indolyphosphate (NBT/BCIP) substrate (Roche) at $37^{\circ} \mathrm{C}$ in the dark for $\sim 3 \mathrm{~h}$. Slides were then rinsed in $1 \times \operatorname{PBS}(3 \times 5 \mathrm{~min})$, fixed in $4 \%$ PFA (10 min), washed with $1 \times$ PBS $(3 \times 5 \mathrm{~min})$, and coverslipped with aquamount media (Thermo-Scientific).

To test for probe specificity, additional brains were collected and prepared as above. A cfos sense control riboprobe was generated by adding the T3 sequence to the forward primer instead of the reverse primer (as in anti-sense probes). One set of slides was stained with sense probe while the alternate set was stained with anti-sense probe and run simultaneously. The sense controls did not show any labeling in the brain (Figure 2).

\section{Quantification of Brain Activation}

To quantify differences in $c f o s$ staining, slides were visualized on a Nikon Eclipse Ni microscope and photographs were taken with a color digital camera controlled by Nikon Elements software. Brightfield and phase contrast were used to visualize neuroanatomical markers and brain nuclei in relation to DIGlabeled cells. A cresyl violet stained A. burtoni reference brain, A. burtoni brain atlas, and other relevant papers (Fernald and Shelton, 1985; Munchrath and Hofmann, 2010; Maruska et al., 2012a) were used for identification of neuroanatomical markers. Images were taken at the highest magnification that encompassed the entire area of interest. The following brain nuclei were analyzed: sensory regions: medial octavolateralis nucleus (MON), ventrolateral portion of the torus semicircularis (TSvl), central portion of the torus semicircularis (TSc; torus semicircularis $=$ putative homolog of the mammalian inferior colliculus), central posterior thalamic nucleus $(\mathrm{CP})$, posterior part of the dorsal telencephalon (Dp; putative homolog of mammalian piriform cortex); SDMN regions: anterior tuberal nucleus (ATn; homolog of ventromedial hypothalamus), central part of the dorsal telencephalon, subdivision 4 (Dc-4), granular zone of the lateral zone of the dorsal telencephalon (Dlg; homolog of medial pallium; hippocampus), magnocellular preoptic nucleus magnocellular division (nMMp), magnocellular preoptic nuclus parvocellular division (nPMp), periventricular nucleus of the posterior tuberculum (TPp; homolog of ventral tegemental area), caudal subdivision of the dorsal part of the ventral telencephalon (Vdc; homologous in part to nucleus accumbens/basal ganglia), supracomissural nucleus of the ventral telencephalon (Vs; homolog of central/medial/extended amygdala), ventral portion of the ventral telencephalon ( $\mathrm{Vv}$; homolog of lateral septum). The medial portion of the dorsal telencephalon (Dm; part of SDMN; homologous in part to pallial amygdala) was not quantified due to low cfos staining in all animals and the inability to reliably distinguish between its subdivisions. Similarly, the periaqueductal gray (PAG; part of SDMN) was not examined because of its small size and inability to distinguish it from surrounding nuclei. The lateral preglomerular nucleus (PGl; receives lateral line input) was also not quantified due to hazy staining instead of discrete cell staining and the inability to distinguish between mechanosensory and other sensory processing subdivisions. Other SDMN nuclei (Vc, central part of the ventral telencephalon [striatum]; VTn, ventral tuberal nucleus [anterior hypothalamus], and Vl, lateral part of the ventral telencephalon) were not quantified for similar reasons.

For nuclei with relatively low cfos-stained cell densities (e.g., $\mathrm{MON}$ ), the region was outlined and the area was measured in Nikon Elements. Individual cells were then counted and cell density (cells $/ \mu \mathrm{m}^{2}$ ) was calculated by dividing the number of cells by the total quantified nucleus area $\left(\mu \mathrm{m}^{2}\right)$. For nuclei with high-density cfos-stained cells (e.g., ATn, CP), the region was outlined and gridlines (either $15 \times 15 \mu \mathrm{m}$, or $50 \times 50$ $\mu \mathrm{m}$ dependent on magnification) were applied to the image. Numbers were assigned to all boxes that were fully encompassed by the outline and a random number generator was used to designate which boxes were used to count cell numbers. Three or five boxes per section were quantified depending on size of the region. Cell density was calculated by dividing the number of cells within the box by the area of the box. For both methods, four consecutive sections were quantified for each region and averaged together for a cell density value of that region in a particular animal. Cell quantification data were then averaged across all individual fish within each group. The entirety of each region was not quantified for every nucleus. All cfos quantification was done blind to fish identity.

\section{Anosmic Controls}

During analysis of $c f o s$ activation in the brain, we noted drastically reduced staining in olfactory processing regions such as the inner cellular layer of the olfactory bulb and the posterior portion of the dorsal telencephalon (Dp) in lateral line-ablated fish, suggesting that $2 \mathrm{mM} \mathrm{CoCl}_{2}$ treatment may also impair chemosensory systems. To ensure that any behavior differences were not due to lack of olfaction, we also included anosmic fish 


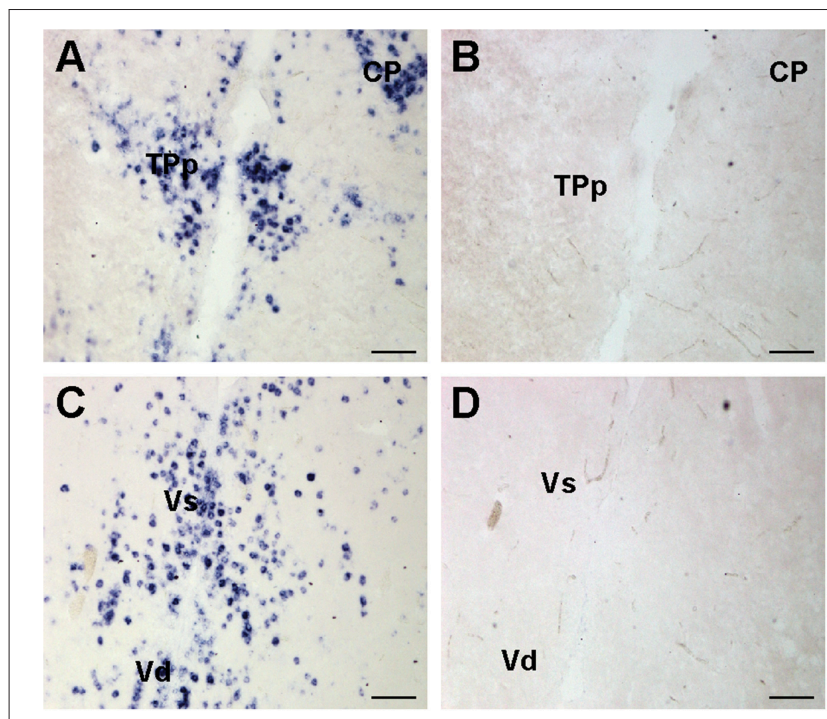

FIGURE 2 | Representative examples of cfos in situ hybridization staining to show probe specificity. Photomicrographs of adjacent transverse sections from the same brain stained with cfos antisense $(\mathbf{A}, \mathbf{C})$ and sense control probes (B,D). Sense control probes showed no staining in any region. Scale bars represent $100 \mu \mathrm{m}$. See list for abbreviations.

as a control in addition to the sham treated controls described above. To create anosmic fish, we used a micro-cauterizer (Cautery High Temp Adjust-A-Temp Fine Tip, Bovie Medical Corporation) to burn off and ablate the olfactory epithelium prior to placing fish in the same experimental paradigm used for our mechanosensory trials $(N=5)$. Fish were allowed to recover for 2 days prior to the forced territorial interaction. Brains from fish in three anosmic-anosmic trials were collected, stained for cfos mRNA, and analyzed as described above.

\section{Statistical Analysis}

Comparisons of GSI, SL, BM and behavior data with only one variable (i.e., intact vs. ablated) were compared with student's $t$-tests in SigmaPlot 12.3. If assumptions could not be met by transforming the data, non-parametric statistics were used and are reported appropriately throughout the text. Because winners and losers came from the same interactions and cannot be considered independent, linear mixed models were used to examine the impact of treatment (intact vs. ablated vs. anosmic) and outcome (winner vs. loser) on behavior data and brain activation data using SPSS 19. Two random effects were used, one for individual subjects and the other for each dyad. Pairwise comparisons with least significant difference (LSD) adjustments were used to determine differences between treatment (intact vs. ablated vs. anosmic). Pearson correlations were used to test for correlations between $c f o s$ staining data of each brain nucleus and other regions and behaviors. Corrections for multiple testing were done by controlling for the false discovery rate (FDR) using the Benjamini-Hochberg procedure with an FDR of 0.25. All significant $p$-values remained significant after FDR corrections for FDR, so only the exact $p$-values and effect size (e.g., $r$-values) are included in each table. We chose not to use Bonferroni corrections or other similar procedures because they reduce statistical power and increase the chance of type II errors, especially in small sample sizes. While these tests do reduce type I errors, their unacceptable effects on statistical power can hide potential biologically relevant results (Nakagawa, 2004). Unless otherwise stated, all animals were combined for correlations and multivariate analysis. Factor analyses were performed in SPSS using principal component extractions with Eigenvalues $>1$. Missing values were excluded listwise and small coefficients $(<0.3)$ were suppressed. Components 1 and 2 were plotted in rotated space (varimax rotation). Only regions of the SDMN and mechanosensory processing regions were included in the multivariate analyses (TSc, Dp, Dc-4 removed). Similarly, only SDMN and mechanosensory processing regions were used in the discriminant function analysis, and missing values were replaced with the group mean for the discriminant function analysis only. All groups were considered equal during the classification, and classification was done using within-group covariance.

\section{RESULTS}

\section{Lateral Line Ablation and Impact on Social Behavior}

To examine territorial behaviors of lateral line-intact and -ablated animals we used a previously described behavioral paradigm in which two dominant $A$. burtoni males have equal opportunity to acquire a novel territory shelter (Figure 1A; Butler and Maruska, 2015). Territorial interactions occurred between two lateral lineintact males ( $N=6$ trials; 12 fish) or two lateral line-ablated males ( $N=6$ trials; 12 fish) and were scored for stereotypical aggressive and assessment behaviors. Lateral line-ablated fish spent significantly more time within one body length of their opponent (Figure 1B; $t$-test; $t=-3.268, d f=10, P=0.008$ ), suggesting a decrease in assessment abilities similar to that previously described (Butler and Maruska, 2015). Latency to fight and fight duration, however, were similar in trials between two lateral line-intact and two lateral line-ablated fish (latency: $t=$ $-0.711, d f=10, P=0.493$; fight time: $t=1.174, d f=10$, $P=0.268$ ). Although aggressive scores (number of aggressive behaviors per minute) were similar for all fish (Figure 1C; LMM; treatment: $F=0.598, P=0.453$; outcome: $F=0.700, P=$ 0.421 ; treatment $\times$ outcome: $F=0.015, P=0.905$ ), lateral line-ablated males relied more on contact aggressive behaviors while lateral line-intact males used predominantly non-contact aggressive behaviors (Figure 1D; treatment: $F=11.766, P=$ 0.005 ; outcome: $F=0.279, P=0.611$; treatment $\times$ outcome: $F=$ $0.017, P=0.900)$. These data indicate that the lateral line system is involved in opponent assessment and the facilitation of noncontact behaviors, as previously reported in A. burtoni (Butler and Maruska, 2015).

\section{Activation of Sensory Processing Brain Regions}

Brains from lateral line-intact and -ablated fight winners and losers were collected following territorial interactions to compare 
cfos expression in nuclei implicated in sensory processing and social decision-making. Mechanosensory cues are detected by neuromasts, the functional units of the lateral line system (Dijkgraaf, 1963), and this hydrodynamic information is sent via the anterior and posterior lateral line nerves into the MON of the hindbrain (McCormick, 1983; Meredith, 1984; Wullimann and Grothe, 2014). Lateral line-ablated animals had decreased cfos staining in the MON compared to lateral line-intact animals (Figures 3A-C; LMM; treatment: $F=24.397, P<0.001$; outcome: $F=1.488, P=0.234$; treatment $\times$ outcome: $F<0.001$, $P=0.996$; post-hoc LSD: $P<0.001$ ) indicating that our ablation method was successful. The MON sends ascending projections to the ventrolateral portion of the midbrain torus semicircularis (TSvl; Luiten, 1975; McCormick, 1982, 1989; Echteler, 1984). The TSvl processes lateral line cues while the central portion of the torus semicircularis (TSc) processes auditory signals. Lateral line-ablated animals had decreased cfos staining in the TSvl (Figures 3D-F; treatment: $F=42.093, P<0.001$; outcome: $F=0.210, P=0.656$; treatment $\times$ outcome: $F=0.125$, $P=0.731$; LSD: $P<0.001$ ) but not the TSc (treatment: $F=$ 0.113, $P=0.893$; outcome: $F=1.731, P=0.221$; treatment $\times$ outcome: $F=0.001, P=0.953)$ indicating that treatment had no impact on audition but further demonstrates impaired mechanoreceptive capabilities. In addition, lateral line-ablated animals had decreased staining in the central posterior thalamic nucleus (CP; Figures 3G-I; treatment: $F=7.374, P=0.006$; outcome: $F=0.259, P=0.619$; treatment $\times$ outcome: $F=0.106$, $P=0.751$; LSD: $P=0.003$ ), which acts as a relay station for both auditory and mechanosensory signals (Finger and Tong, 1984). Since the TSc was not different between intact and ablated
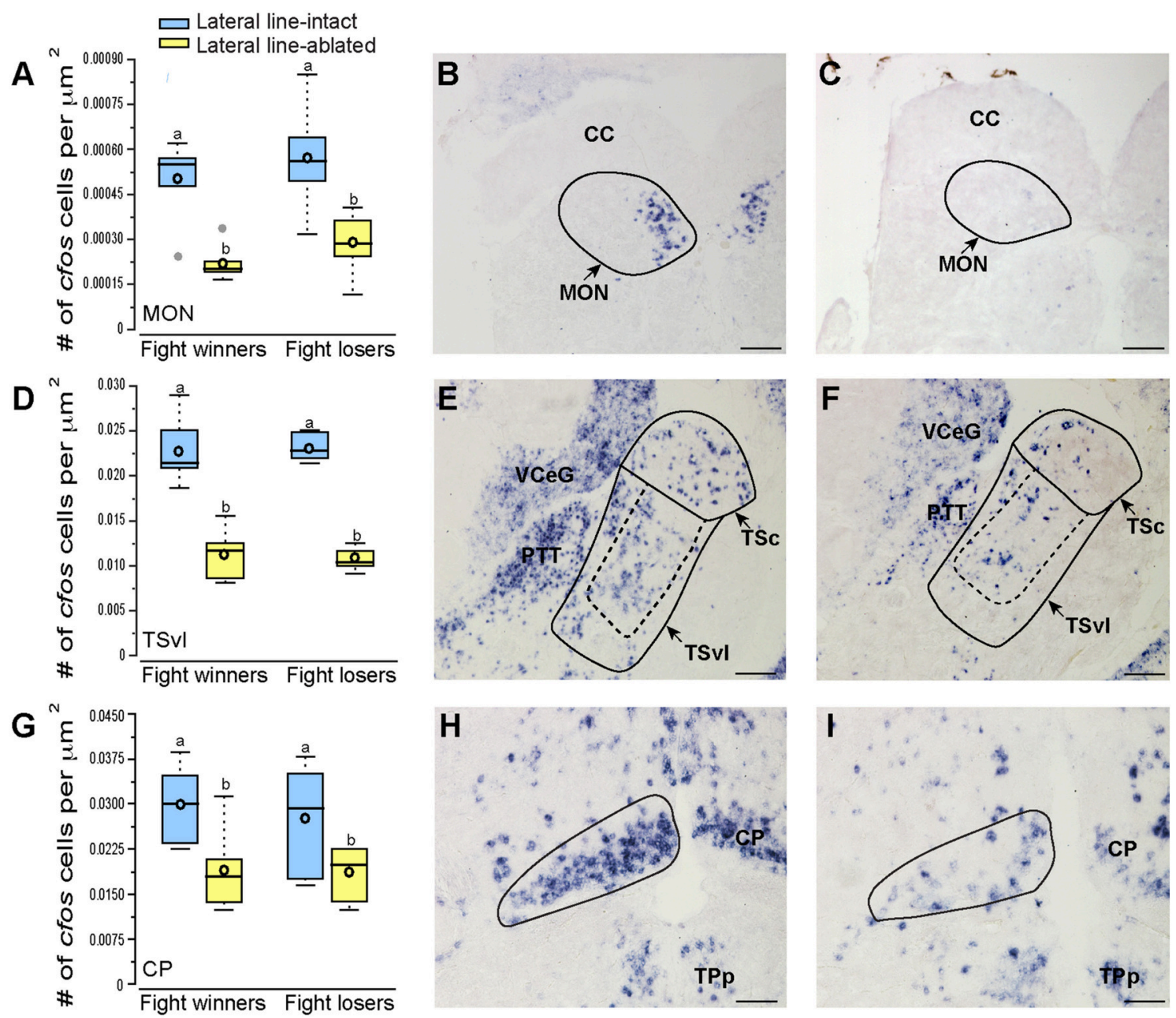

FIGURE 3 | Lateral line-ablated animals have decreased cfos staining in lateral line processing regions of the brain. Quantification of cfos staining in the MON (A), TSvl (D), and CP (G) in lateral line-intact (blue) and -ablated (yellow) fight winners and losers ( $N=6$ for all groups). Photomicrographs of representative cfos staining (dark blue label) in transverse sections through the MON of a lateral line-intact (B) and -ablated (C) animal, the TSvl of an intact (E) and ablated (F) animal, and the CP of an intact $\mathbf{( H )}$ and ablated $\mathbf{( I )}$ animal. Solid circles depict nuclei borders and dashed outlines represent the subarea quantified for each nucleus. If no dashed outline is present, the entire nucleus was quantified. Different letters indicate statistical significance at $P<0.05$ using post-hoc LSD tests. Scale bars in (B,C,E,F) represent $100 \mu \mathrm{m}$. Scale bars in $\mathbf{( H , I ) ~ r e p r e s e n t ~} 25 \mu \mathrm{m}$. See Figure 1 legend for box plot descriptions. See list for abbreviations. 
animals, the difference in activation of the $\mathrm{CP}$ is likely due to decreased mechanosensory processing in lateral line-ablated fish.

\section{Activation of Socially-Relevant Brain Regions}

To examine which brain regions process socially-relevant hydrodynamic cues, we quantified cfos expression in regions of the SDMN. Lateral line-ablated fish had decreased staining in the anterior tuberal nucleus (ATn; Figures $4 \mathrm{~A}-\mathrm{C}$; treatment: $F=13.511, P<0.001$; outcome: $F=1.758, P=0.212$; treatment $\times$ outcome: $F=3.119, P=0.106$; LSD: $P<0.001)$, supracommissural nucleus of the ventral telencephalon (Vs; Figures 4D-F; treatment: $F=5.852, P=0.010$; outcome: $F=$ 1.581, $P=0.226$; treatment $\times$ outcome: $F=0.682, P=0.421$; LSD: P0.004), and the granular zone of the lateral zone of the dorsal telencephalon (Dlg; Figures 4G-I; treatment: $F=12.115$,
$P<0.001$; outcome: $F=0.176, P=0.679$; treatment $\times$ outcome: $F=0.140, P=0.713$; LSD: $P=0.001)$, but none were affected by fight outcome (i.e., winning or losing). In contrast, lateral lineintact and -ablated animals had similar $c f o s$ staining in the ventral part of the ventral telencephalon (Vv; Figures 5A-C; treatment: $F=1.213, P=0.33$; outcome: $F=2.121, P=0.176$; treatment $\times$ outcome: $F=0.004, P=0.948)$, the periventricular nucleus of the posterior tuberculum (TPp; Figures 5D-E; treatment: $F=$ 1.493, $P=0.256$; outcome: $F=1.036, P=0.330$; treatment $\times$ outcome: $F=0.546, P=0.475$ ), and the caudal subdivision of the dorsal part of the ventral telencephalon (Vdc; treatment: $F$ $=0.272, F=0.765$; outcome: $F=1.187, P=0.289$; treatment $\times$ outcome: $F=0.046, P=0.823$ ). We quantified two different parts of the preoptic area (POA), the nMMp (magnocellular preoptic nucleus, magnocellular division) and the nPMp (magnocellular preoptic nucleus, parvocellular division). There was no effect
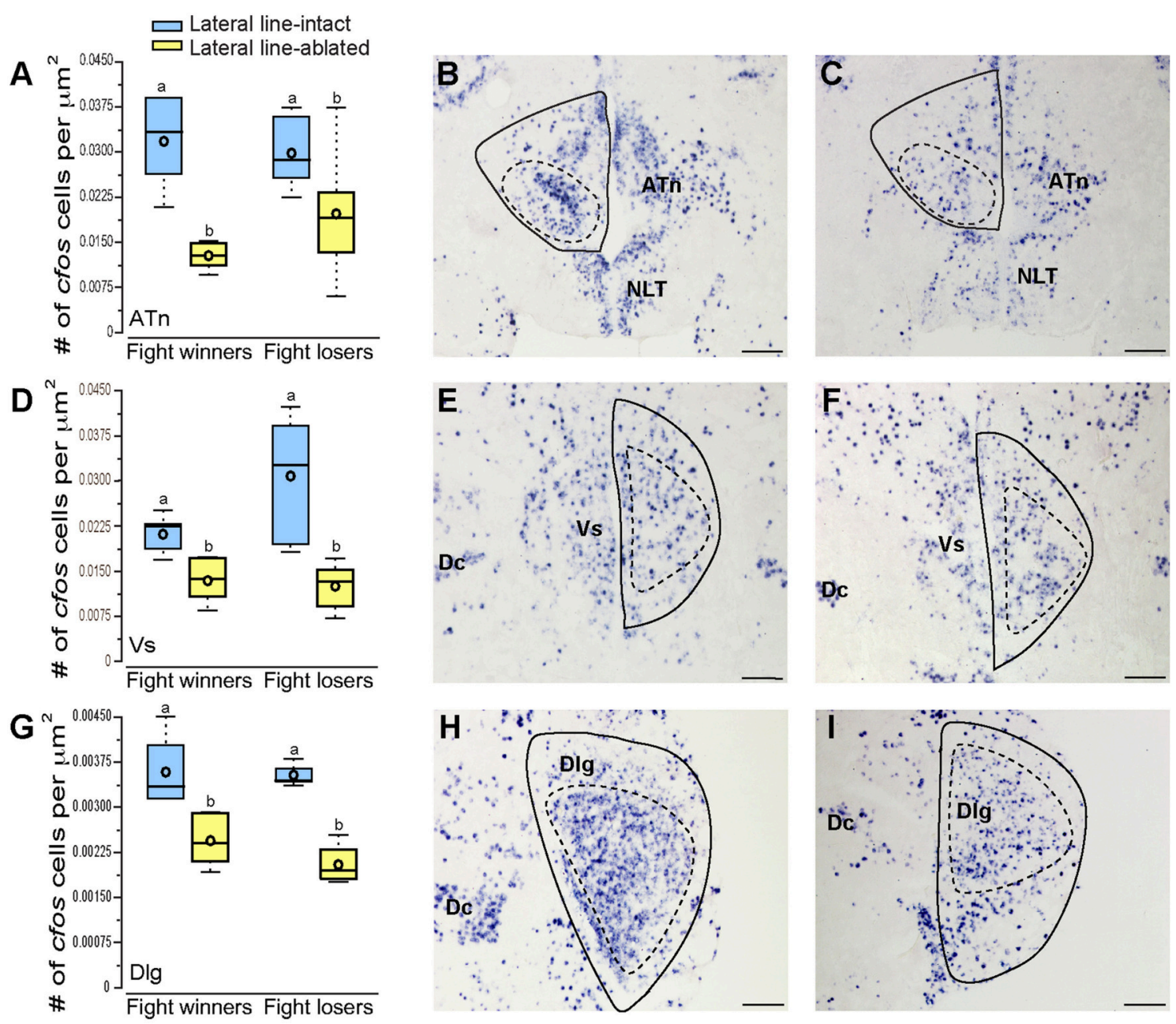

FIGURE 4 | Lateral line-ablated animals have decreased cfos staining in several SDMN nuclei. Quantification of cfos staining in the ATn (A), Vs (D), and Dlg (G) in lateral line-intact (blue) and -ablated (yellow) fight winners and losers ( $N=6$ for all groups). Photomicrographs of representative cfos staining (dark blue label) in transverse sections through the ATn of a lateral line-intact (B) and -ablated (C) animal, the Vs of an intact (E) and ablated (F) animal, and the Dlg of an intact $(\mathbf{H})$ and ablated (I) animal. Solid circles depict nuclei borders and dashed outlines represent the subarea quantified for each nucleus. Different letters indicate statistical significance at $P<0.05$ using post-hoc LSD tests. Scale bars in (B, C,E, F, H, I) represent $100 \mu \mathrm{m}$. See Figure $\mathbf{1}$ legend for box plot descriptions. See list for abbreviations. 

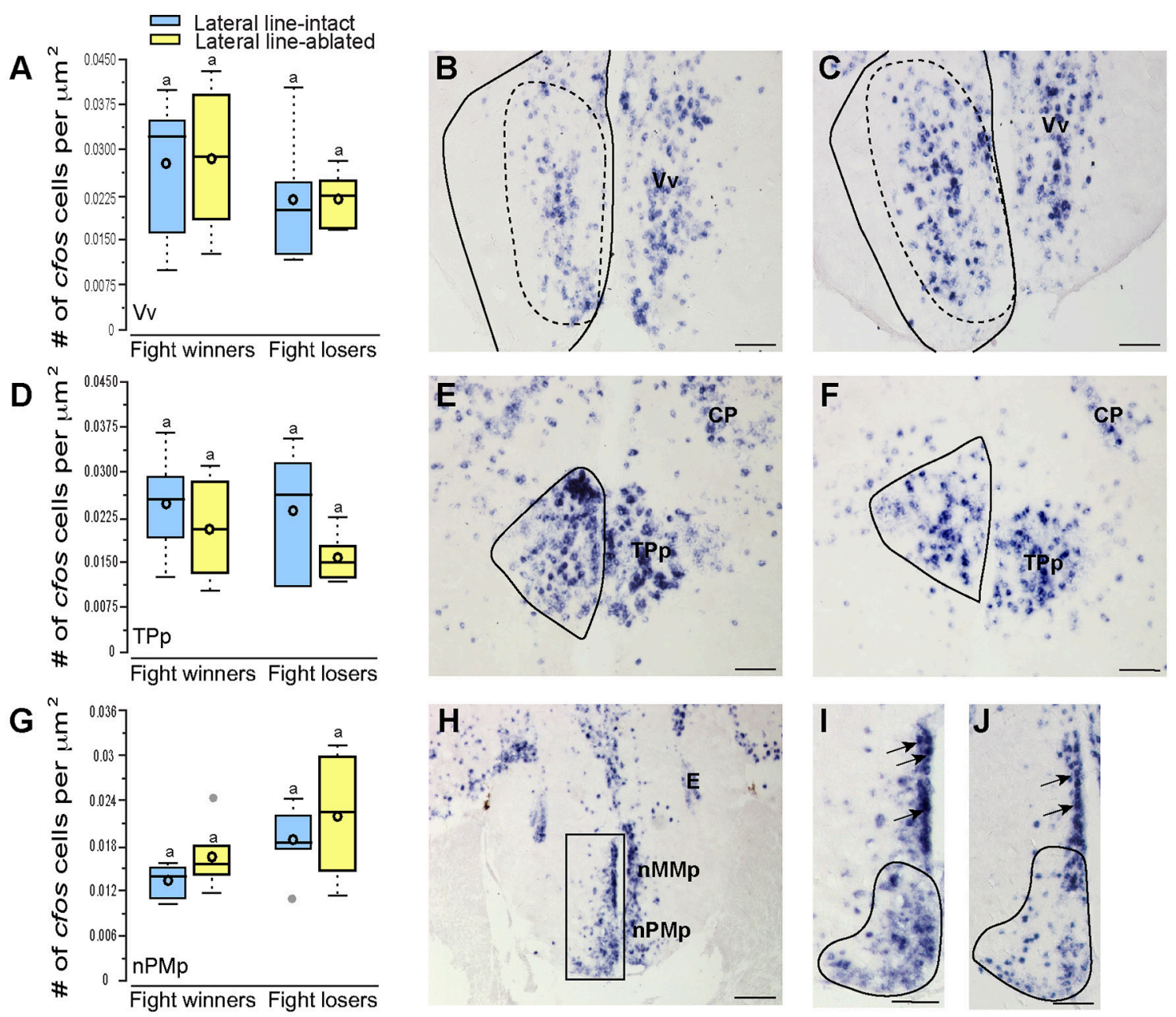

FIGURE 5 | Fight losers have increased activation of the nPMp but other SDMN regions are unaffected. Quantification of cfos staining in the VV (A), TPp (D), and nPMp (G) in lateral line-intact (blue) and -ablated (yellow) fight winners and losers ( $N=5-6$ for all groups). Photomicrographs of representative cfos staining (dark blue label) in the $\mathrm{V} v$ of a lateral line-intact (B) and -ablated (C) animal, and the TPp of an intact (E) and ablated (F) animal. The preoptic area was divided into two parts, the nMMp, and the nPMp for quantification (H). Representative photomicrographs in the POA of lateral line-intact fight winner $\mathbf{( I )}$ and loser $(\mathbf{J})$. Arrows in $\mathbf{( I , J )}$ indicate magnocellular cells quantified as part of the nMMp. Solid circles depict nuclei borders and dashed outlines represent the subarea quantified for each nucleus, If no dashed outline is present, the entire nucleus was quantified. Different letters indicate statistical significance at $P<0.05$ using post-hoc LSD tests. Scale bars in (B,C,E,F) represent $25 \mu \mathrm{m}$. Scale bars in $\mathbf{( H )}$ represents $100 \mu \mathrm{m}$ and scales in $\mathbf{( I , J ) ~ r e p r e s e n t ~} 12.5 \mu \mathrm{m}$. See Figure 1 legend for box plot descriptions. See list for abbreviations.

of treatment or fight outcome on $c f o s$ staining in the nMMp (treatment: $F=0.566, P=0.578$; outcome: $F=1.610, P=$ 0.233 ; treatment $\times$ outcome: $F=1.396, P=0.264$ ) or the $\mathrm{nPMp}$ (Figures 5G-J; treatment: $F=0.062, P=0.940$; outcome: $F=$ $1.119, P=0.316$; treatment $\times$ outcome: $F=0.400, P=0.537)$. These data indicate that parts of the SDMN (ATn, Vs, Dlg) likely receive and process socially-relevant hydrodynamic cues while activation of other nuclei is independent of mechanosensory input, at least in this aggressive territorial context.

\section{Activation of Brain Regions in Anosmic Controls}

Noticeably reduced DASPEI (vital dye used to verify lateral line ablation) staining of the olfactory epithelium following lateral line ablation suggested that cobalt chloride treatment also impaired olfaction (Butler and Maruska, 2015). To examine this, we compared cfos staining in the posterior portion of the dorsal telencephalon (Dp), a region known to process olfactory information in fishes (Satou, 1990) (Figure 6). Lateral lineablated animals had reduced staining in the $\mathrm{Dp}$ (treatment: $F=4.992, P=0.024$; outcome: $F=0.056, P=0.816$; treatment $\times$ outcome: $F=0.949, \quad P=0.349$; LSD: $P$ $=0.012$ ), but lateral line-ablated and anosmic fish have similar cfos staining in the Dp (LSD: $P=0.570$ ). Thus, we included anosmic (i.e., olfactory epithelium ablated) animals in behavior trials and compared cfos staining of anosmic fight winners to lateral line-intact fight winners to ensure that any observed differences were due to impairment of the lateral line system and not comorbid effects on chemosensory systems. 


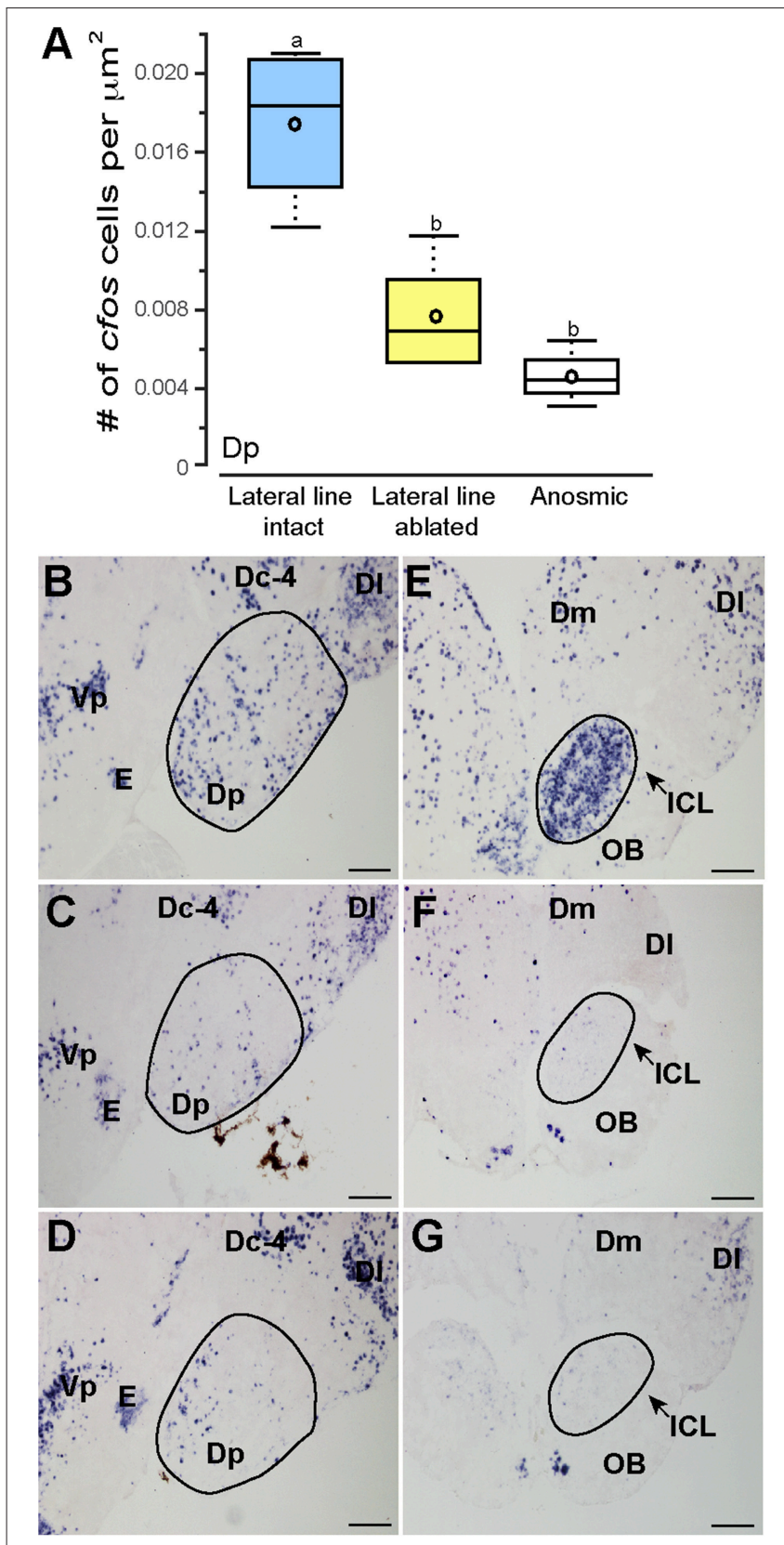

FIGURE 6 | Cobalt chloride treatment impaired olfactory processing in lateral line-ablated fish. Quantification of cfos staining in the known

telencephalic olfactory processing region, Dp (A) of lateral line-intact (blue; $N$ =6), lateral line-ablated (yellow; $N=5$ ), and anosmic (white; $N=3$ ) fight winners and corresponding photomicrographs of representative cfos staining in the Dp of a lateral line-intact (B) -ablated (C), and anosmic (D) animal, and the olfactory bulbs of an intact (E), ablated (F), and anosmic (G) animal. Circled regions represent the area quantified for each nucleus. Different letters indicate statistical significance at $P<0.05$ using post-hoc LSD tests. Scale bars in (B-G) represent $100 \mu \mathrm{m}$. See Figure 1 legend for box plot descriptions. See list for abbreviations.

Lateral line-intact and anosmic fight winners had similar cfos staining in all previously mentioned brain regions (MON, TSvl, TSc, CP, ATn, Vs, Dlg, Vv, TPp, Vdc, nMMp, and nPMp; $P>$
TABLE 1 | Effect of treatment and outcome on cfos expression in sensory and socially-relevant brain regions.

\begin{tabular}{|c|c|c|c|c|c|c|}
\hline & \multicolumn{2}{|c|}{ Treatment } & \multicolumn{2}{|c|}{ Outcome } & \multicolumn{2}{|c|}{ Treatment x Outcome } \\
\hline & $\boldsymbol{F}$ & $\boldsymbol{P}$ & $\boldsymbol{F}$ & $\boldsymbol{P}$ & $\boldsymbol{F}$ & $P$ \\
\hline MON & 24.397 & $<0.001$ & 1.488 & 0.234 & $<0.001$ & 0.996 \\
\hline TSvl & 42.093 & $<0.001$ & 0.210 & 0.656 & 0.125 & 0.731 \\
\hline CP & 7.374 & 0.006 & 0.259 & 0.619 & 0.106 & 0.750 \\
\hline ATn & 13.511 & $<0.001$ & 1.758 & 0.212 & 3.119 & 0.106 \\
\hline Vs & 5.852 & 0.010 & 1.581 & 0.226 & 0.682 & 0.421 \\
\hline Dlg & 12.115 & $<0.001$ & 0.176 & 0.679 & 0.140 & 0.713 \\
\hline TPp & 1.493 & 0.256 & 1.036 & 0.330 & 0.546 & 0.475 \\
\hline nMMp & 0.566 & 0.578 & 1.610 & 0.233 & 1.396 & 0.264 \\
\hline nPMp & 0.062 & 0.940 & 1.119 & 0.316 & 0.400 & 0.537 \\
\hline VV & 1.213 & 0.333 & 2.121 & 0.176 & 0.004 & 0.948 \\
\hline Vdc & 0.272 & 0.765 & 1.187 & 0.289 & 0.046 & 0.823 \\
\hline TSc & 0.113 & 0.893 & 1.731 & 0.221 & 0.001 & 0.953 \\
\hline Dp & 4.992 & 0.024 & 0.056 & 0.816 & 0.949 & 0.349 \\
\hline Dc-4 & 3.609 & 0.050 & 1.039 & 0.336 & 0.002 & 0.964 \\
\hline
\end{tabular}

\begin{tabular}{lccc}
\hline \multicolumn{4}{c}{ Multiple comparisons (treatment) } \\
\hline & Sham-Ablated & Sham-Anosmic & Ablated-Anosmic \\
\hline & $\boldsymbol{P}$ & $\boldsymbol{P}$ \\
\hline MON & $<\mathbf{0 . 0 0 1}$ & 0.950 & $\mathbf{0 . 0 0 1}$ \\
TSvl & $<\mathbf{0 . 0 0 1}$ & 0.781 & $\mathbf{0 . 0 0 1}$ \\
CP & $\mathbf{0 . 0 0 3}$ & 0.884 & $\mathbf{0 . 0 2 3}$ \\
ATn & $<\mathbf{0 . 0 0 1}$ & 0.722 & $\mathbf{0 . 0 0 2}$ \\
Vs & $\mathbf{0 . 0 0 4}$ & 0.812 & $\mathbf{0 . 0 1 8}$ \\
Dlg & $\mathbf{0 . 0 0 1}$ & 0.482 & $\mathbf{0 . 0 0 2}$ \\
TPp & 0.109 & 0.923 & 0.308 \\
nMMp & 0.543 & 0.571 & 0.923 \\
nPMp & 0.742 & 0.557 & 0.380 \\
Vv & 0.908 & 0.266 & 0.242 \\
Vdc & 0.491 & 0.759 & 0.909 \\
TSc & 0.947 & 0.908 & 0.873 \\
Dp & $\mathbf{0 . 0 1 2}$ & 0.059 & 0.570 \\
Dc-4 & 0.129 & $\mathbf{0 . 0 0 9}$ & 0.133 \\
\hline & & & \\
\hline & & & \\
\hline
\end{tabular}

Main effects, interactions, and multiple comparisons were calculated using linear mixed models. Bold indicates significance at $P<0.05$.

0.05 for all, see Table 1 for statistics), suggesting that impaired olfaction did not contribute to cfos staining differences in these regions between lateral line-intact and -ablated fish. Lateral lineablated and anosmic animals had higher cfos staining in the central part of the dorsal telencephalon, subdivision 4 (Dc-4; treatment: $F=3.609, P=0.050$; outcome: $F=1.039, P=0.336$; treatment $\times$ outcome: $F=0.002, P=0.964$; LSD: $P=0.009$ ) compared to lateral-line-intact fish. Because this difference is partly a result of impaired olfaction, Dc-4 was removed from multivariate analyses. 

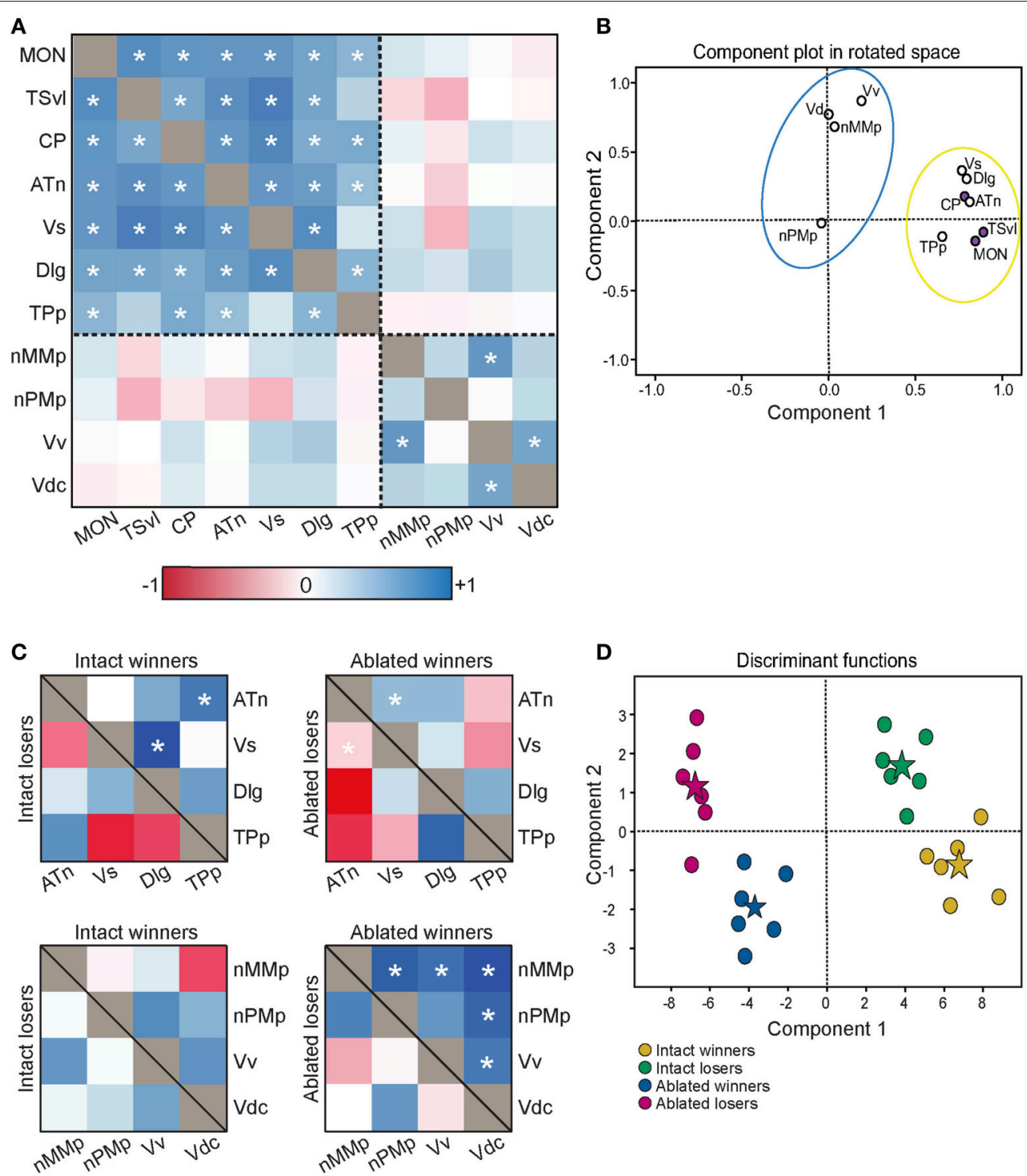

FIGURE 7 | Lateral line-ablated animals have altered co-activation of functional networks within the SDMN. (A) Heat map of Pearson correlation coefficients $(R=$ color scale) of cfos staining in brain nuclei correlated with other nuclei ( $N=24$ animals per region; See also Table 2). Dashed lines separate the two functional networks. (B) Principal component analysis of cfos staining in sensory processing (purple dots) and social behavior (white dots) brain nuclei reveal two distinct networks. Dashed circle encompasses network that incorporates mechanosensory cues, and solid circle encompasses second network without lateral line input. (C) Heat maps of Pearson correlation coefficients of two functional networks within the SDMN ( $N=6$ animals per group). Top two boxes represent network 1 (SDMN nuclei that receive lateral line input) with winners above the black line and losers below. Bottom two boxes represent network 2 (other SDMN nuclei). (D) Discriminant function analysis distinguished lateral line-intact fight winners (yellow) and losers (green) from lateral line-ablated fight winners (blue) and losers (pink). Stars represent each group centroid. ${ }^{*} P<0.05$ on heat maps. See list for abbreviations.

\section{Correlations and Multivariate Analyses of Multiple Brain Regions}

To determine the functional connectivity of the nodes of the SDMN, we first correlated cfos staining across brain regions (Figure 7A, Table 2). Lateral line processing regions (MON, TSvl, and CP) all positively correlated with each other. In addition, the ATn, Vs, and Dlg positively correlated with each other and with lateral line processing regions, providing further evidence that they process mechanosensory information. Cfos staining in the $\mathrm{Vv}$ positively correlated with the nMMp and the $\mathrm{Vdc}$. There were no correlations between lateral line processing regions and the nMMp, nPMp, $\mathrm{Vv}$, or $\mathrm{Vdc}$ suggesting that activation of these regions is not mediated by mechanosensory input in this agonistic context. Principal component analysis (PCA) of cfos activation in lateral line processing and SDMN nuclei produced two significant components describing the variability in the data (Figure 7B; Table 3; $N=24$ animals; Kaiser-Meyer-Olkin measure of sampling adequacy $=0.505$; 
TABLE 2 | Pearson correlation values of cfos staining in each brain nucleus correlated with all other brain nuclei.

\begin{tabular}{|c|c|c|c|c|c|c|c|c|c|c|c|c|c|c|}
\hline & & TSvl & CP & ATn & Vs & Dlg & TPp & nMMp & nPMp & Vv & Vdc & TSc & Dp & Dc-4 \\
\hline \multirow[t]{2}{*}{ MON } & $\mathrm{R}$ & 0.694 & 0.650 & 0.644 & 0.647 & 0.573 & 0.476 & 0.181 & 0.122 & -0.031 & -0.074 & 0.237 & 0.682 & -0.524 \\
\hline & $P$ & $<0.001$ & $<0.001$ & $<0.001$ & $<0.001$ & 0.006 & 0.019 & 0.408 & 0.578 & 0.886 & 0.738 & 0.265 & $<0.001$ & 0.012 \\
\hline \multirow[t]{2}{*}{ TSvl } & $\mathrm{R}$ & & 0.554 & 0.686 & 0.765 & 0.563 & 0.305 & -0.149 & -0.277 & 0.010 & -0.043 & 0.184 & 0.789 & -0.676 \\
\hline & $P$ & & 0.006 & $<0.001$ & $<0.001$ & 0.010 & 0.158 & 0.508 & 0.211 & 0.963 & 0.850 & 0.401 & $<0.001$ & $<0.001$ \\
\hline \multirow[t]{2}{*}{$\mathrm{CP}$} & $\mathrm{R}$ & & & 0.634 & 0.778 & 0.528 & 0.552 & 0.103 & -0.093 & 0.215 & 0.147 & 0.110 & 0.528 & -0.350 \\
\hline & $P$ & & & $<0.001$ & $<0.001$ & 0.014 & 0.005 & 0.639 & 0.672 & 0.314 & 0.503 & 0.610 & 0.014 & 0.110 \\
\hline \multirow[t]{2}{*}{ ATn } & $\mathrm{R}$ & & & & 0.647 & 0.609 & 0.440 & -0.028 & -0.176 & 0.020 & 0.037 & 0.132 & 0.581 & -0.339 \\
\hline & $P$ & & & & $<0.001$ & 0.003 & 0.031 & 0.898 & 0.421 & 0.923 & 0.867 & 0.540 & 0.006 & 0.123 \\
\hline \multirow[t]{2}{*}{ Vs } & $\mathrm{R}$ & & & & & 0.696 & 0.177 & 0.217 & -0.268 & 0.309 & 0.245 & 0.305 & 0.817 & -0.423 \\
\hline & $P$ & & & & & $<0.001$ & 0.418 & 0.331 & 0.228 & 0.151 & 0.273 & 0.157 & $<0.001$ & 0.056 \\
\hline \multirow[t]{2}{*}{ Dlg } & $\mathrm{R}$ & & & & & & 0.487 & 0.246 & 0.190 & 0.356 & 0.244 & 0.349 & 0.757 & -0.149 \\
\hline & $P$ & & & & & & 0.025 & 0.217 & 0.410 & 0.113 & 0.287 & 0.120 & $<0.001$ & 0.543 \\
\hline \multirow[t]{2}{*}{ TPp } & $\mathrm{R}$ & & & & & & & -0.044 & -0.053 & -0.036 & 0.020 & 0.040 & 9.279 & -0.174 \\
\hline & $P$ & & & & & & & 0.842 & 0.811 & 0.867 & 0.929 & 0.852 & 0.220 & 0.439 \\
\hline \multirow[t]{2}{*}{ nMMp } & $\mathrm{R}$ & & & & & & & & 0.267 & 0.636 & 0.304 & 0.438 & 0.100 & 0.144 \\
\hline & $P$ & & & & & & & & 0.217 & 0.001 & 0.170 & 0.037 & 0.673 & 0.534 \\
\hline \multirow[t]{2}{*}{ nPMp } & $\mathrm{R}$ & & & & & & & & & 0.028 & 0.261 & 0.003 & -0.216 & 0.232 \\
\hline & $P$ & & & & & & & & & 0.901 & 0.241 & 0.987 & 0.361 & 0.312 \\
\hline \multirow[t]{2}{*}{ Vv } & $\mathrm{R}$ & & & & & & & & & & 0.563 & 0.566 & 0.215 & 0.415 \\
\hline & $P$ & & & & & & & & & & 0.005 & 0.004 & 0.349 & 0.055 \\
\hline \multirow[t]{2}{*}{ Vdc } & $\mathrm{R}$ & & & & & & & & & & & 0.153 & 0.195 & 0.385 \\
\hline & $P$ & & & & & & & & & & & 0.485 & 0.398 & 0.085 \\
\hline \multirow[t]{2}{*}{ TSc } & $\mathrm{R}$ & & & & & & & & & & & & 0.310 & 0.328 \\
\hline & $P$ & & & & & & & & & & & & 0.171 & 0.136 \\
\hline \multirow[t]{2}{*}{ Dp } & $\mathrm{R}$ & & & & & & & & & & & & & -0.555 \\
\hline & $P$ & & & & & & & & & & & & & 0.014 \\
\hline
\end{tabular}

Pearson correlation values $(R)$ were used to create the heat map in Figure 7A. Bold indicates significance at $P<0.050$.

Bartlett's test of sphericity chi-squared $=122.741, d f=66, P<$ 0.001 ). The first component (42.489\% of variance) was strongly weighted by the MON, TSvl and ATn, and the second component (17.533\% of variance explained) was weighted primarily by the $\mathrm{Vv}$ and nMMp. Based on the nodes driving each component, component 1 most likely represents mechanosensory input and component 2 likely represents social behavior regions relating to reproduction and social status. Based on these correlations and PCA, we identified two distinct functional networks within the SDMN. The first consists of the ATn, Vs, Dlg, and TPp, and the second consists of the Vv, Vd, nMMp, and nPMp. In the context of this territorial behavioral paradigm, these two functional networks likely represent two distinct networks within the SDMN: one that receives mechanosensory signals to mediate behavioral output, and the other that acts independent of mechanosensory input.

To compare activation and connectivity of these functional networks in lateral line-intact and -ablated fight winners and losers, we used Pearson correlations and created heat maps based on the correlation coefficients (Figure 7C). While few significant correlations were detected (likely due to small sample sizes; $N=6$ for each group), heat maps allow for better visualization of the differences. The most striking difference occurs in lateral line-ablated fight winners. No other group had significant correlations (i.e., co-activity) of the functional network containing the $\mathrm{Vv}, \mathrm{Vd}, \mathrm{nMMp}$, and nPMp; however, lateral line-ablated fight winners had strong co-activity of all regions within this network and little co-activity within the functional network containing mechanosensory-mediated brain nuclei. These data indicate that in the absence of mechanosensory input, activation of functional networks within the SDMN is altered.

To further examine these differences in network activation, we next used canonical discriminant function analysis (CDA; Figure 7D; Table 3). Discriminant function analysis combines all input variables into a single composite score and can identify which variables (i.e., brain regions) contribute to differentiation between the four animal groups. CDA verifies that animals can be distinguished via brain activation alone and can provide insight into which nodes of the SDMN are driving these distinctions. Our discriminant function analysis accurately predicted $100 \%$ of ablated winners, $83.33 \%$ of ablated losers, but only $50 \%$ of lateral line-intact fish, and there was no cross over between lateral line-intact and -ablated animals. Furthermore, all of these values exceed classification by chance (25\%). Component 1 was driven by the MON, TSvl, and the Vs, and explained $84.6 \%$ of the variance in the data. Component 2 was loaded primarily by the TSvl, MON, nMMp, nPMp, ATn, Vd, and Vv, and only explained 
$14 \%$ of the data, and component 3 was equally loaded by all brain regions and only accounted for $<2 \%$ of the variance. Together, the first 2 components explained more than $98 \%$ of the variance in the data and clearly separated the four animal groups based on brain activation alone.

\section{Correlation of Behavior and Brain Activation Data}

To further understand the role of the SDMN in processing socially-relevant mechanosensory signals, we correlated fight behaviors with brain cfos activation data (Figure 8A; Table 4). Latency of fight time negatively correlated only with $c f o s$ activation in the CP $(R=-0.497, P=0.014)$, while fight duration positively correlated with the MON $(R=0.404, P=$ $0.047)$. Assessment time negatively correlated with the number of cfos-labeled cells in both the Vs $(R=-0.432, P=0.039)$ and the $\operatorname{Dlg}(R=-0.537, P=0.012)$ and the number of prefight behaviors positively correlated with the TSvl, CP, and Vs ( $R>0.40, P<0.05$ for each). In addition, behavioral preference (ratio of contact to non-contact behaviors) negatively correlated with $c f o s$ activation in all three lateral line processing regions, the ATn, and the Vs $(R<-0.40, P<0.05$ for each $)$, but aggressive scores did not correlate with any quantified regions. The nMMp, nPMp, Vv, and Vd did not correlate with any fight behaviors. We also correlated the three discriminant function scores of each animal to their behaviors (Figure 8B). Function 1 (loaded primarily by mechanosensory-mediated brain regions) negatively correlated with behavioral preference $(R=-0.618$, $P<0.001)$ and assessment time $(R=-0.406, P=0.049)$, and positively correlated with the number of pre-fight aggressive behaviors ( $R=0.478, P=0.018)$. Functions 2 and 3, however, did not correlate with any measured fight behaviors.

To further examine the relationship between fight behaviors and activation of sensory and socially-relevant brain regions, we ran a PCA on brain and behavior data together (Figure 8C; Table 3). This analysis pulled out three main components $(N=$ 24 animals; Kaiser-Meyer-Olkin measure of sampling adequacy $=0.301$; Bartlett's test of sphericity chi-squared $=207.794$, $d f=136, P<0.001)$. The first consisting primarily of lateral line processing regions, along with their correlated behaviors (behavioral choice, assessment abilities, and number of prefight behaviors), and this component explained $34.224 \%$ of variance. Component 2 was loaded primarily by the TPp and fight duration (13.558\% variance explained), and component 3 was loaded by the nMMp, nPMp Vv, Vdc, aggressive score, and latency to fight (10.959\% variance explained). The correlation of assessment behaviors and behavioral choice with regions of the SDMN and functional network co-activity further indicates the crucial role of mechanosensory cues in mediating these behaviors.

\section{DISCUSSION}

We provide for the first time in any fish species evidence of where socially-relevant mechanosensory information is processed in the brain (Figure 9). After identifying nuclei that receive and
TABLE 3 | Loading values for multivariate analyses.

\begin{tabular}{|c|c|c|c|}
\hline \multicolumn{4}{|c|}{ PCA (Figure 7B) component loadings } \\
\hline & Component 1 & Component 2 & Component 3 \\
\hline MON & 0.770 & -0.318 & 0.370 \\
\hline TSvl & 0.842 & -0.329 & -0.168 \\
\hline $\mathrm{CP}$ & 0.812 & -0.053 & -0.040 \\
\hline ATn & 0.829 & -0.089 & -0.058 \\
\hline Vs & 0.851 & 0.118 & -0.229 \\
\hline Dlg & 0.826 & 0.106 & 0.217 \\
\hline Vv & 0.425 & 0.780 & -0.237 \\
\hline Vdc & 0.201 & 0.719 & -0.090 \\
\hline TPp & 0.593 & -0.268 & 0.106 \\
\hline nMMp & 0.205 & 0.690 & 0.361 \\
\hline nPMp & -0.072 & 0.096 & 0.921 \\
\hline \multicolumn{4}{|c|}{ CDA (Figure 7D) factor loadings } \\
\hline & Factor 1 & Factor 2 & Factor 3 \\
\hline MON & 0.183 & 0.376 & 0.071 \\
\hline TSvl & 0.419 & 0.425 & 0.185 \\
\hline $\mathrm{CP}$ & 0.132 & 0.073 & -0.072 \\
\hline ATn & 0.168 & 0.314 & -0.340 \\
\hline Vs & 0.208 & 0.118 & -0.011 \\
\hline Dlg & 0.151 & 0.103 & -0.124 \\
\hline Vv & 0.010 & -0.207 & -0.017 \\
\hline Vdc & 0.013 & -0.239 & -0.261 \\
\hline TPp & 0.072 & -0.059 & 0.129 \\
\hline nMMp & 0.008 & -0.112 & 0.142 \\
\hline nPMp & -0.081 & 0.277 & 0.044 \\
\hline
\end{tabular}

\begin{tabular}{lccc}
\hline \multicolumn{4}{c}{ PCA (Figure 8C) component loading } \\
\hline & Component 1 & Component 2 & Component 3 \\
\hline MON & $\mathbf{0 . 7 9 3}$ & - & - \\
TSvl & $\mathbf{0 . 8 6 9}$ & - & - \\
ATn & $\mathbf{0 . 8 0 6}$ & - & - \\
TPp & $\mathbf{0 . 5 6 6}$ & - & - \\
CP & $\mathbf{0 . 8 1 8}$ & - & - \\
VS & $\mathbf{0 . 8 4 8}$ & - & - \\
nMMp & - & $\mathbf{0 . 8 3 4}$ & - \\
nPMp & - & - & 0.398 \\
Dlg & $\mathbf{0 . 7 9 1}$ & - & - \\
VV & 0.323 & $\mathbf{0 . 6 5 7}$ & 0.315 \\
Vdc & - & 0.346 & $\mathbf{0 . 7 0 9}$ \\
BehaviorChoice & $-\mathbf{0 . 6 7 4}$ & - & 0.483 \\
Aggression & - & 0.470 & $-\mathbf{0 . 6 9 0}$ \\
Latency & - & -0.396 & - \\
Fight & 0.460 & - & 0.384 \\
Assessment & $-\mathbf{0 . 5 2 6}$ & - & 0.419 \\
Pre-fight & 0.553 & 0.556 & - \\
& & & \\
\hline & & &
\end{tabular}

Component loadings for principle component analyses and discriminant function analysis. Bold indicates the highest loading factors ( $>0.5$ for PCA and $>0.2$ for $C D A)$. 

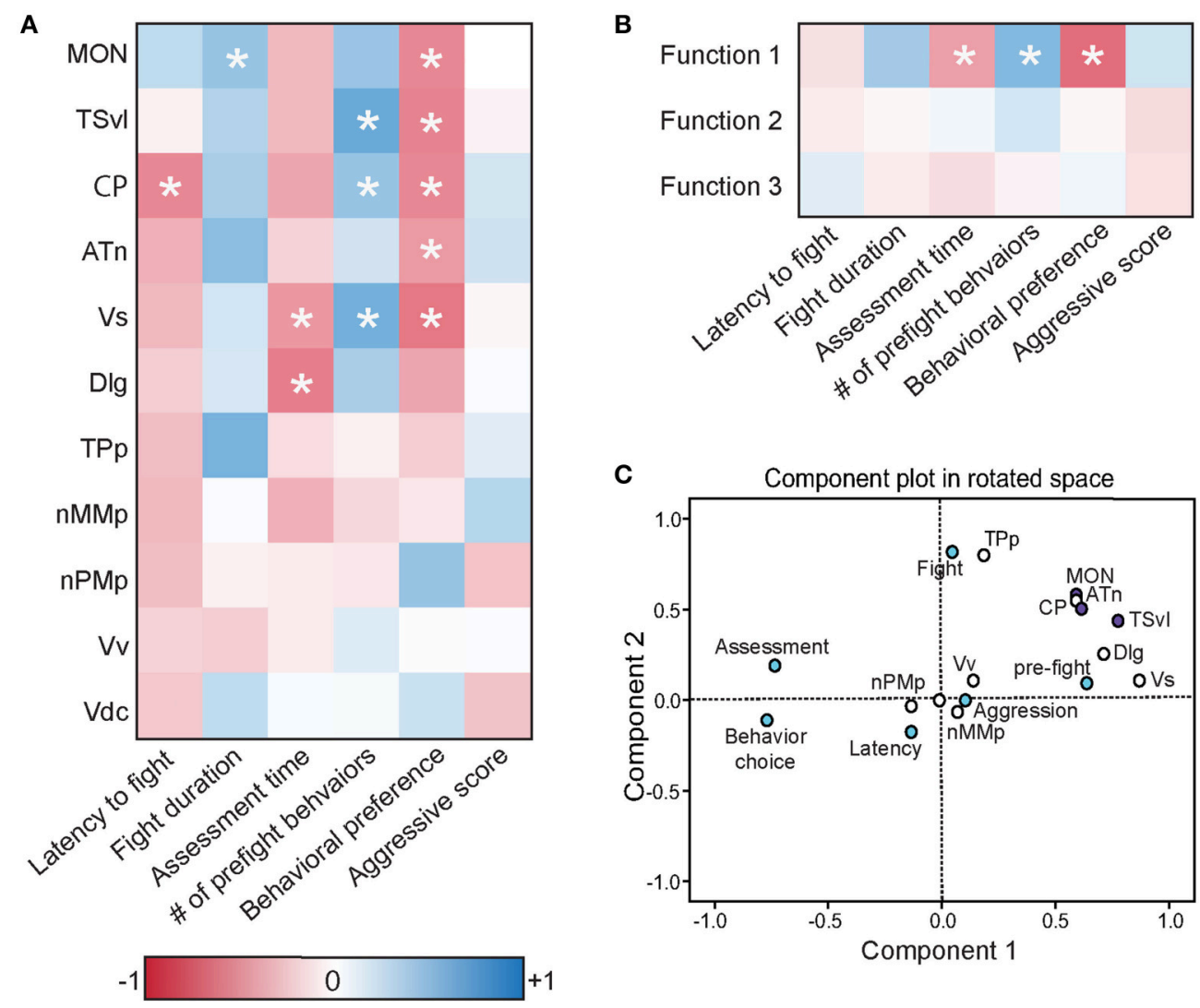

FIGURE 8 | Brain cfos staining correlates with fight and assessment behaviors. (A) Heat map of Pearson correlation coefficients ( $R=$ color scale) of cfos staining in brain nuclei with behaviors (see also Table 4). (B) Heat map of Pearson correlation coefficients of the CDA functions with behaviors. Function 1 (loaded primarily by the MON, TSvl, ATn, Dlg, and Vs) separated lateral line-intact and -ablated animals. Function 2 (loaded primarily by the TSvl, MON, nMMp, nPMp, ATn, Vdc, and Vv) separated fight winners and losers. Function 3 was equally loaded by all brain regions. (C) Principal component analysis of cfos staining in sensory processing regions (purple dots), social behaviors (white dots), and fight and assessment behaviors (blue dots). ${ }^{*}<<0.05$. See list for abbreviations. Aggression: aggressive score during territorial interaction. Assessment: \% of prefight time spent within one body length of opponent. Behavior choice: ratio of contact to non-contact fight behaviors. Fight: fight duration. Latency: latency to fight. Pre-fight: number of unreciprocated prefight aggressive behaviors.

process mechanosensory signals, we used a network activity approach to identify two distinct functional networks. During male-male territorial interactions, one network consisting of the $\mathrm{ATn}, \mathrm{Vs}, \mathrm{Dlg}$, and TPp receives and processes mechanosensory signals to modify behavioral output while the second network consisting of the $\mathrm{Vv}, \mathrm{Vdc}, \mathrm{nMMp}$, and $\mathrm{nPMp}$ acts independent of mechanosensory input. These data provide novel insight into how mechanosensory cues are integrated into brain networks controlling social behaviors to produce context-appropriate behavioral responses.

\section{Impact of Cobalt Chloride Treatment on Sensory Processing}

We first examined $c f o s$ staining in sensory processing brain nuclei of lateral line-ablated and -intact fight winners and losers to verify that cobalt chloride impaired reception of mechanosensory cues. While previous studies have used neural tract-tracing and electrophysiological recording techniques in anesthetized fish to elucidate some of the lateral line processing regions in the brain (reviewed in Wullimann and Grothe, 2014), our work is the first to identify mechanosensory processing regions in naturally behaving animals with and without lateral line input. Lateral line-ablated fish had decreased cfos staining (indicating decreased activation) in all examined lateral line processing regions (MON, TSvl, CP). Treatment efficacy was initially verified via reduced DASPEI staining of neuromasts (Butler and Maruska, 2015), and reduced cfos staining in lateral line brain regions further demonstrates that our $\mathrm{CoCl}_{2}$ treatment functionally disabled neuromasts of the mechanosensory lateral line system. $\mathrm{CoCl}_{2}$ treatment reduced evoked activity in the posterior lateral line nerve of the roach Rutilus rutilus (Karlsen and Sand, 1987), but there is a paucity of studies verifying $\mathrm{CoCl}_{2}$ treatment efficacy beyond neuromast vital dye staining. All lateral lineablated animals had some positive cfos staining in lateral line processing regions, suggesting that ablation may not have been $100 \%$. It is possible that the observed cfos staining in lateral lineablated animals was due to remaining functional neuromasts, spontaneous activity in the anterior lateral line nerves, or activation via circuits from other nuclei. 
TABLE 4 | Correlations of fight and assessment behaviors with brain cfos quantification data.

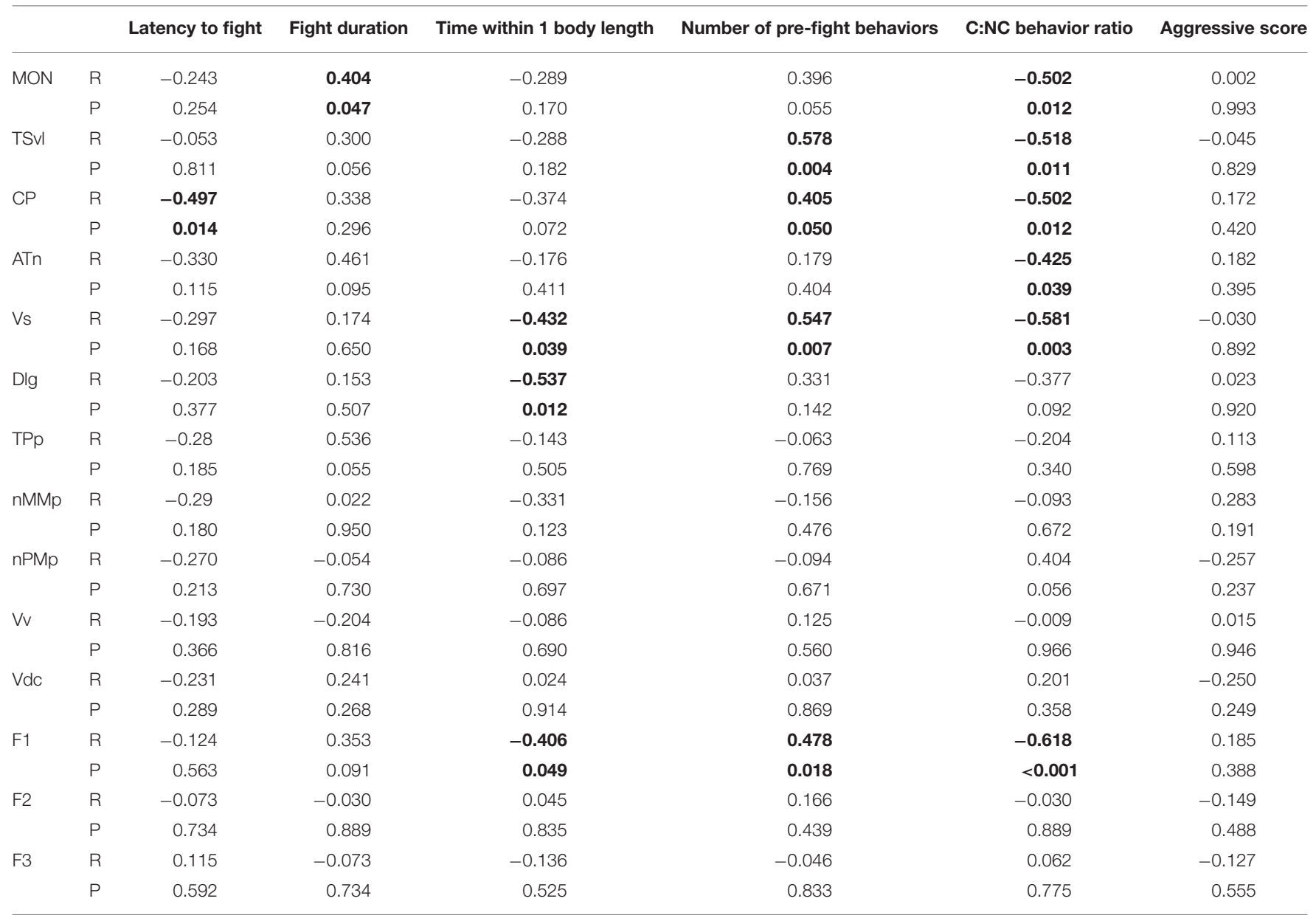

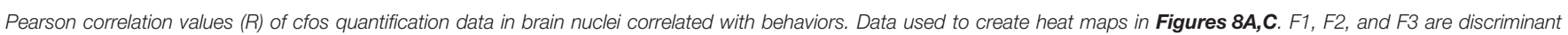
scores of each animal for functions 1, 2, and 3. Bold indicates significance at $P<0.050$.

We also examined cfos staining in central processing regions of auditory and olfactory signals to look for comorbid effects on other sensory systems. Karlsen and Sand (1987) previously showed that cobalt treatment had no impact on hearing capabilities (measured via utricular microphonic potentials) in $R$. rutilus, but because of the higher dose used in our experiments and the species variability of $\mathrm{CoCl}_{2}$ treatments, we also quantified cfos staining in the auditory TSc. We found no difference between lateral line-intact and -ablated animals in the TSc, indicating that our treatment had no impact on hair cells of the inner ear. We previously found that fish treated with $2 \mathrm{mM} \mathrm{CoCl} 2$ had noticeably reduced staining in the olfactory epithelium (Butler and Maruska, 2015), suggesting an impact on olfaction. In addition, $\mathrm{CoCl}_{2}$ treated animals had decreased cfos staining in olfactory processing regions (e.g., olfactory bulb and Dp) indicating that cobalt chloride also impairs sensory cells of the olfactory epithelium in addition to neuromasts of the lateral line system (and although not tested, cobalt likely also impacts taste buds and solitary chemosensory cells located on the surface of fish). A. burtoni, like many teleost fishes, use multimodal signaling during social behaviors, and males use chemosensory signaling during male-male social interactions (Maruska and Fernald, 2012). Because of this, we included anosmic (olfactory epithelium ablated) animals in our study and compared behavior and brain activation of anosmic winners to lateral line-intact winners. These data and controls allowed us to be confident that any observed behavioral and $c f o s$ expression differences were due solely to loss of mechanoreceptive capabilities and not olfaction.

\section{Central Processing of Socially-Relevant Mechanosensory Cues}

The social decision making-network was first proposed as an integrative network incorporating the highly connected SBN and mesolimbic reward system (O'Connell and Hofmann, 2011). Together, these networks are thought to be involved in mediating adaptive (i.e., rewarding) social behaviors by evaluating the salience of a sensory cue and integrating it with the animals own internal physiology to produce an appropriate behavioral response. We first determined which nuclei of the SDMN receive mechanosensory cues by examining cfos staining differences in each brain region, and then examined how co-activation of functional networks within the SDMN are influenced by 


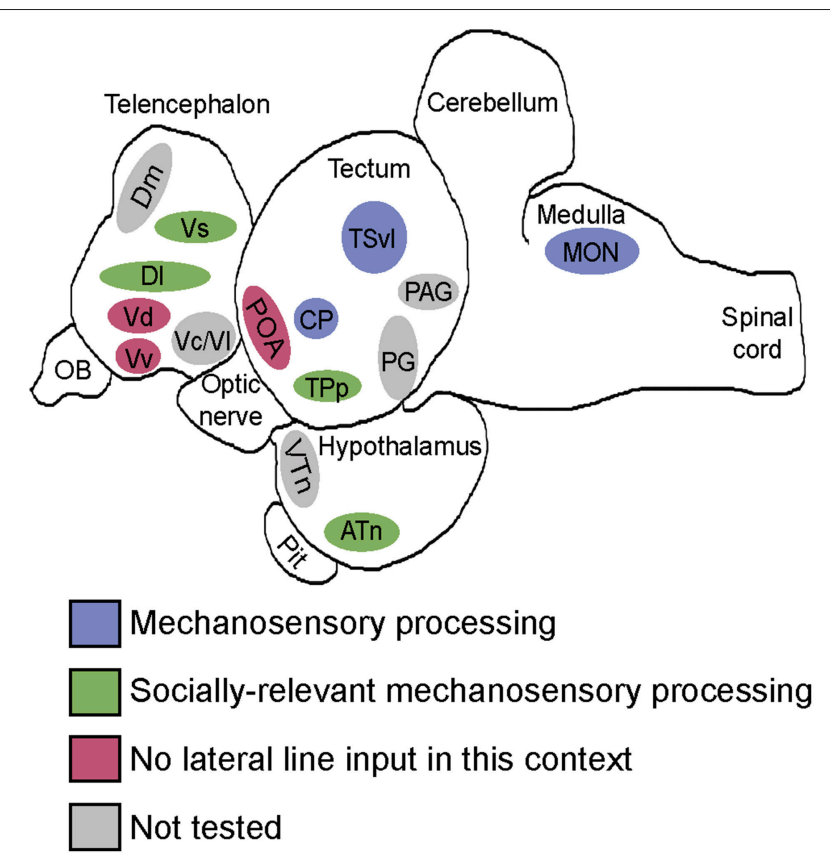

FIGURE 9 | Summary schematic of socially-relevant mechanosensory processing in the cichlid brain. Blue represents mechanosensory processing regions. Green represents nuclei of the SDMN that receive lateral line input and possibly use it to modify behavioral output. Pink represents SDMN nuclei without evidence for mechanosensory input during aggressive contexts. Gray represents SDMN and sensory processing regions not tested for a potential role in processing socially-relevant mechanosensory signals. Locations of nuclei on the sagittal brain outline are only approximate. See list for abbreviations.

mechanosensory cues. The ATn receives direct and indirect input from the torus semicircularis (TS) and is considered by some to be an octavolateralis nucleus because of these connections (Striedter, 1991; Wullimann and Grothe, 2014). It is, therefore, an ideal candidate for linking mechanosensory information with other brain nuclei implicated in social decision-making. Although the ventromedial hypothalamus (VMH; mammalian homolog of the ATn) plays an important role in mediating aggressive and reproductive behaviors in mammals (Olivier, 1977; Kollack-Walker and Newman, 1995; Lin et al., 2011; Yang et al., 2013) there is little evidence of the functional role of the ATn in teleost fishes. The ATn expresses sex-steroid receptors (Munchrath and Hofmann, 2010), is activated following social encounters, and in A. burtoni, is likely involved in social status transitions (Maruska et al., 2013). In A. burtoni, the ATn processes socially-relevant mechanosensory cues, as indicated by the decreased $c f o s$ staining in the ATn of lateral line-ablated animals and the negative correlation of cfos staining in the ATn with behavioral choice (contact vs. non-contact behaviors). It is possible, therefore, that mechanosensory cues could influence behavioral choice, social interactions, and social status transitions via the ATn and its connections.

The ATn and other lateral line processing regions project directly to several forebrain nuclei of the SDMN, such as the Dm, Dl, and Dc (Yamamoto and Ito, 2008; Wullimann and Grothe,
2014). Lateral line-ablated animals had reduced staining in the Vs and Dlg compared to lateral line-intact fish indicating that both regions receive hydrodynamic input. In addition, both regions negatively correlated with assessment time, and cfos staining in the Vs positively correlated with the number of unreciprocated pre-fight aggressive behaviors and negatively with the animal's behavioral preference. These correlations suggest that not only do these regions receive lateral line input, but they may also be involved in animal assessment and behavioral choices. Several tract-tracing studies have demonstrated connections between mechanosensory processing regions in pallial brain nuclei (Dl, Dc, Dm), but this is the first evidence of a subpallial nucleus (Vs) receiving mechanosensory information. The Vs is thought to be homologous in part to the mammalian medial extended amygdala (O'Connell and Hofmann, 2011), which is known for its role in incorporating sensory cues (Turner and Herkenham, 1991; Gray, 1999) to modify behaviors, and it is possible that the Vs has a similar role in the teleost brain.

\section{Co-activation of Functional Networks of the SDMN Depends on Mechanosensory Input}

When first describing the SBN, Newman proposed that specific behavioral states (e.g., male sexual behavior) are most likely due to the pattern of activation across nodes of the SBN rather than at one specific node (Newman, 1999; Yang and Wilczynski, 2007; Teles et al., 2015). To examine this, we first sought to determine the functional networks within the SDMN in the context of socially-relevant hydrodynamic cues. Correlation heat maps and PCA identified two distinct networks. The first consisted of lateral line sensory processing regions, regions of the SDMN that receive lateral line input, and the TPp. This network was primarily loaded by the MON, TSvl, CP, and ATn indicating that it is dependent on lateral line input. The inclusion of SDMN regions (ATn, Vs, Dlg, and TPp) with sensory processing regions (MON, TSvl, CP) in this network provides further evidence for mechanosensory input to these nuclei. The second network consisted of the remaining SDMN nuclei ( $\mathrm{Vv}, \mathrm{Vdc}, \mathrm{nMMp}$, and nPMp). These nuclei have functions related to reproduction and social status, and activation of these regions appears to be independent of mechanosensory input. In the context of male-male agonistic interactions, it is possible that the first component (or network) is closely tied to the fight itself while the second network is more closely related to winning an agonistic interaction and acquiring a spawning territory (i.e., reproductive fitness and status position consequences). Analyses of co-activity of these functional networks in lateral line-intact and -ablated fight winners and losers revealed distinct activation patterns. For example, lateral line-intact fight winners had significant, positive co-activity of the network containing the Vs, Dlg, ATn, and TPp and no significant co-activity of the second network, but lateral line-ablated fight winners had little co-activation of the first network and strong co-activation of the second. One hypothesis is that the first network (receiving lateral line input) is dominating the output of the SDMN when mechanoreceptive capabilities are intact, but without mechanosensory input, 
activation of the SDMN is driven more by the fight outcome itself (i.e., acquiring a spawning territory). These differences in connectivity and activation across functional networks indicate that socially-relevant hydrodynamic cues are essential to the SDMN.

The role of interconnected neural networks rather than individual nuclei is likely also applicable to complex social behaviors such as opponent assessment. Animal assessment is extensively studied via behavioral analyses (reviewed in Arnott and Elwood, 2009), but little is known about which brain regions are involved in opponent mutual assessment. Because mutual assessment is dependent on integration of multimodal sensory information, it is likely that assessment behaviors are dependent on a network of nuclei working together to integrate sensory cues with the animal's own internal physiology. Our data indicate that during male-male territorial disputes, assessment abilities correlate the co-activity of mechanosensory processing regions and the ATn, Vs, Dlg, and TPp. This novel result further supports the notion that mechanosensory cues are used to assess opponents and provides the first evidence for where in the teleost brain this mutual assessment may occur.

\section{CONCLUSIONS}

Here we show for the first time that socially-relevant hydrodynamic cues are processed in nodes of the SDMN and that without lateral line input, fish have altered activation of functional networks within the SDMN. Furthermore, lateral line-ablated fish alter their behavioral choices and have decreased assessment ability, which is related to activation of these functional networks in the brain. Although the role of visual, chemosensory, and auditory cues are well-studied for their role(s) in social behaviors, our data indicates that the mechanosensory lateral line system is an integral part of social

\section{REFERENCES}

Arnott, G., and Elwood, R. W. (2009). Assessment of fighting ability in animal contests. Anim. Behav. 77, 991-1004. doi: 10.1016/j.anbehav.2009.02.010

Butler, J. M., and Maruska, K. P. (2015). The mechanosensory lateral line is used to assess opponents and mediate aggressive behaviors during territorial interactions in an African cichlid fish. J. Exp. Biol. 218, 3284-3294. doi: $10.1242 /$ jeb. 125948

Chen, C.-C., and Fernald, R. D. (2011). Visual information alone changes behavior and physiology during social interactions in a cichlid fish (Astatotilapia burtoni). PLoS ONE 6:e20313. doi: 10.1371/journal.pone.0020313

Dijkgraaf, S. (1963). The functioning and significance of the lateral-line organs. Biol. Rev. Camb. Philos. Soc. 38, 51-105. doi: 10.1111/j.1469185X.1963.tb00654.x

Echteler, S. M. (1984). Connections of the auditory midbrain in a teleost fish, Cyprinus carpio. J. Comp. Neurol. 230, 536-551. doi: 10.1002/cne.902300405

Fernald, R. D. (1977). Quantitative behavioural observations of Haplochromis burtoni under semi-natural conditions. Anim. Behav. 25(Pt 3), 643-653. doi: 10.1016/0003-3472(77)90115-4

Fernald, R. D., and Hirata, N. R. (1977). Field study of Haplochromis burtoni: quantitative behavioural observations. Anim. Behav. 25, 964-975. doi: 10.1016/0003-3472(77)90048-3 interactions and mediates activation of brain regions used in social decisions. Given that many social interactions among the $>30,000$ different species of fishes produce hydrodynamic cues that can be detected by the lateral line system, a better understanding of how this mechanosensory information is integrated in the brain to produce context-dependent behaviors also has broad implications for the evolution of SDMN function across vertebrates.

\section{AUTHOR CONTRIBUTIONS}

Both authors had full access to the data and take full responsibility for the integrity of the data analysis and approved the final manuscript. JB and KM designed experiments. JB performed experiments and analyzed data. JB and KM wrote the manuscript. KM provided funding, equipment, and supplies.

\section{FUNDING}

Funding was provided by startup funds from the College of Science and Department of Biological Sciences at Louisiana State University (KM), Louisiana Board of Regents RCS Grant (KM), Powe Faculty Enhancement Award from ORAU (KM), Sigma Xi (JB), and a Raney Award from ASIH (JB). JB was supported by a Louisiana Board of Regents Graduate Fellowship and National Science Foundation Graduate Research Fellowship.

\section{ACKNOWLEDGMENTS}

We thank Polly Gwan for help with lateral line ablation, members of the Maruska lab for conversations on the data and manuscript, and reviewers for helpful comments on the manuscript.

Fernald, R. D., and Shelton, L. C. (1985). The organization of the diencephalon and the pretectum in the cichlid fish, Haplochromis burtoni. J. Comp. Neurol. 238, 202-217. doi: 10.1002/cne. 902380207

Finger, T. E., and Tong, S.-L. (1984). Central organization of eighth nerve and mechanosensory lateral line systems in the brainstem of ictalurid catfish. $J$. Comp. Neurol. 229, 129-151. doi: 10.1002/cne.902290110

Goodson, J. L. (2005). The vertebrate social behavior network: evolutionary themes and variations. Horm. Behav. 48, 11-22. doi: 10.1016/j.yhbeh.2005. 02.003

Gray, T. S. (1999). Functional and anatomical relationships among the amygdala, basal forebrain, ventral striatum, and cortex: an integrative discussion. Ann. N.Y. Acad. Sci. 877, 439-444. doi: 10.1111/j.1749-6632.1999.tb 09281.x

Karlsen, H. E., and Sand, O. (1987). Selective and reversible blocking of the lateral line in freshwater. Fish. J. Exp. Biol. 133, 249-262.

Kollack-Walker, S., and Newman, S. (1995). Mating and agonistic behavior produce different patterns of Fos immunolabeling in the male Syrian hamster brain. Neuroscience 66, 721-736. doi: 10.1016/0306-4522(94)00 $563-\mathrm{K}$

Lin, D., Boyle, M. P., Dollar, P., Lee, H., Lein, E. S., Perona, P., et al. (2011). Functional identification of an aggression locus in the mouse hypothalamus. Nature 470, 221-226. doi: 10.1038/nature09736 
Luiten, P. G. M. (1975). The central projections of the trigeminal, facial and anterior lateral line nerves in the carp (Cyprinus carpio L.). J. Comp. Neurol. 160, 399-417. doi: 10.1002/cne.901600308

Maruska, K. P. (2014). Social regulation of reproduction in male cichlid fishes. Gen. Comp. Endocrinol. 207, 2-12. doi: 10.1016/j.ygcen.2014.04.038

Maruska, K. P., Becker, L., Neboori, A., and Fernald, R. D. (2013). Social descent with territory loss causes rapid behavioral, endocrine and transcriptional changes in the brain. J. Exp. Biol. 216, 3656-3666. doi: 10.1242/jeb.0 88617

Maruska, K. P., Carpenter, R. E., and Fernald, R. D. (2012a). Characterization of cell proliferation throughout the brain of the African cichlid fish Astatotilapia burtoni and its regulation by social status. J. Comp. Neurol. 520, 3471-3491. doi: $10.1002 /$ cne. 23100

Maruska, K. P., and Fernald, R. D. (2012). Contextual chemosensory urine signaling in an African cichlid fish. J. Exp. Biol. 215, 68-74. doi: 10.1242/jeb.062794

Maruska, K. P., and Fernald, R. D. (2013). Social regulation of male reproductive plasticity in an African cichlid fish. Integr. Comp. Biol. 53, 938-950. doi: 10.1093/icb/ict017

Maruska, K. P., Ung, U. S., and Fernald, R. D. (2012b). The African cichlid fish Astatotilapia burtoni uses acoustic communication for reproduction: sound production, hearing, and behavioral significance. PLOS ONE 7:e37612. doi: 10.1371/journal.pone.0037612

McCormick, C. A. (1982). The organization of the octavolateralis area in actinopterygian fishes: a new interpretation. J. Morph. 171, 159-181. doi: 10.1002/jmor.1051710205

McCormick, C. A. (1983). Central connection of the octavolateralis nerves in the pike cichlid, Crenicichla lepidota. Brain Res. 265, 177-185. doi: 10.1016/00068993(83)90331-1

McCormick, C. A. (1989). "Central lateral line mechanosensory pathways in bony fish," in The Mechanosensory Lateral Line, eds. S. Coombs, H. Bleckmann, R. R. Fay, and A. N. Popper (New York, NY: Springer), 341-364.

Medina, L., Garcia, C., Urbina, A., Manjarrez, J., and Moyaho, A. (2013). Female vibration discourages male courtship behaviour in the Amarillo fish (Girardinichthys multiradiatus). Behav. Processes 100, 163-168. doi: 10.1016/j.beproc.2013.09.007

Meredith, G. E. (1984). Peripheral configuration and central projections of the lateral line system in Astronotus ocellatus (Cichlidae): a nonelectroreceptive teleost. J. Comp. Neurol. 228, 342-358. doi: 10.1002/cne.902280305

Mirjany, M., Preuss, T., and Faber, D. S. (2011). Role of the lateral line mechanosensory system in directionality of goldfish auditory evoked escape response. J. Exp. Biol. 214, 3358-3367. doi: 10.1242/jeb.052894

Montgomery, J., Bleckmann, H., and Coombs, S. (2014). "Sensory ecology and neuroethology of the lateral line," in The Lateral Line System, eds. S. Coombs, H. Bleckmann, R. R. Fay, and A. N. Popper (New York, NY: Springer), 121-150.

Munchrath, L. A., and Hofmann, H. A. (2010). Distribution of sex steroid hormone receptors in the brain of an African cichlid fish, Astatotilapia burtoni. J. Comp. Neurol. 518, 3302-3326. doi: 10.1002/cne.22401

Nakagawa, S. (2004). A farewell to Bonferroni: the problems of low statistical power and publication bias. Behav. Ecol. 15, 1044-1045. doi: 10.1093/beheco/arh107
Newman, S. W. (1999). The medial extended amygdala in male reproductive behavior a node in the mammalian social behavior network. Ann. N.Y. Acad. Sci. 877, 242-257. doi: 10.1111/j.1749-6632.1999.tb09271.x

O'Connell, L. A., and Hofmann, H. A. (2011). The Vertebrate mesolimbic reward system and social behavior network: A comparative synthesis. J. Comp. Neurol. 519, 3599-3639. doi: 10.1002/cne.22735

Olivier, B. (1977). The ventromedial hypothalamus and aggressive behaviour in rats. Aggress. Behav. 3, 47-56. doi: 10.1002/1098-2337(1977)3:1<47::AIDAB2480030105>3.0.CO;2-H

Satou, M. (1990). Synaptic organization, local neuronal circuitry, and functional segregation of the teleost olfactory bulb. Prog. Neurobiol. 34, 115-142. doi: 10.1016/0301-0082(90)90004-Z

Satou, M., Takeuchi, H. A., Nishii, J., Tanabe, M., Kitamura, S., Okumoto, N., et al. (1994). Behavioral and electrophysiological evidences that the lateral line is involved in the inter-sexual vibrational communication of the himé salmon (landlocked red salmon, Oncorhynchus nerka). J. Comp. Physiol. A 174, 539-549. doi: 10.1007/BF00217373

Striedter, G. F. (1991). Auditory, electrosensory, and mechanosensory lateral line pathways through the forebrain in channel catfishes. J. Comp. Neurol. 312, 311-331. doi: 10.1002/cne.903120213

Teles, M. C., Almeida, O., Lopes, J. S., and Oliveira, R. F. (2015). Social interactions elicit rapid shifts in functional connectivity in the social decision-making network of zebrafish. Proc. Biol. Sci. 282:20151099. doi: 10.1098/rspb.2015.1099

Turner, B. H., and Herkenham, M. (1991). Thalamoamygdaloid projections in the rat: a test of the amygdala's role in sensory processing. J. Comp. Neurol. 313, 295-325. doi: 10.1002/cne.903130208

Wullimann, M., and Grothe, B. (2014). "The central nervous organization of the lateral line system," in The Lateral Line System, eds. S. Coombs, H. Bleckmann, R. R. Fay, and A. N. Popper (New York: Springer), 195-251.

Yamamoto, N., and Ito, H. (2008). Visual, lateral line, and auditory ascending pathways to the dorsal telencephalic area through the rostrolateral region of the lateral preglomerular nucleus in cyprinids. J. Comp. Neurol. 508, 615-647. doi: $10.1002 / \mathrm{cne} .21717$

Yang, C. F., Chiang, M. C., Gray, D. C., Prabhakaran, M., Alvarado, M., Juntti, S. A., et al. (2013). Sexually dimorphic neurons in the ventromedial hypothalamus govern mating in both sexes and aggression in males. Cell 153, 896-909. doi: 10.1016/j.cell.2013.04.017

Yang, E.-J., and Wilczynski, W. (2007). Social experience organizes parallel networks in sensory and limbic forebrain. Dev. Neurobiol. 67, 285-303. doi: 10.1002/dneu.20347

Conflict of Interest Statement: The authors declare that the research was conducted in the absence of any commercial or financial relationships that could be construed as a potential conflict of interest.

Copyright (c) 2016 Butler and Maruska. This is an open-access article distributed under the terms of the Creative Commons Attribution License (CC BY). The use, distribution or reproduction in other forums is permitted, provided the original author(s) or licensor are credited and that the original publication in this journal is cited, in accordance with accepted academic practice. No use, distribution or reproduction is permitted which does not comply with these terms. 